\title{
A QUANTITATIVE CENTRAL LIMIT THEOREM FOR THE EULER-POINCARÉ CHARACTERISTIC OF RANDOM SPHERICAL EIGENFUNCTIONS ${ }^{1}$
}

\author{
By VALENTINA CAMmarota AND DOMENICO MARINUCCI
}

Sapienza University of Rome and University of Rome Tor Vergata

We establish here a quantitative central limit theorem (in Wasserstein distance) for the Euler-Poincaré characteristic of excursion sets of random spherical eigenfunctions in dimension 2. Our proof is based upon a decomposition of the Euler-Poincaré characteristic into different Wiener-chaos components: we prove that its asymptotic behaviour is dominated by a single term, corresponding to the chaotic component of order two. As a consequence, we show how the asymptotic dependence on the threshold level $u$ is fully degenerate, that is, the Euler-Poincaré characteristic converges to a single random variable times a deterministic function of the threshold. This deterministic function has a zero at the origin, where the variance is thus asymptotically of smaller order. We discuss also a possible unifying framework for the Lipschitz-Killing curvatures of the excursion sets for Gaussian spherical harmonics.

1. Introduction. The Euler-Poincaré characteristic is perhaps the single most important tool for the analysis of excursion sets for Gaussian random fields; classical textbooks on its behaviour are [1,2], while some very recent contributions can be found for instance in $[10,11,14,21,31]$. As is well known, the Euler-Poincaré characteristic, which we shall denote by $\chi(\cdot)$, is the unique integer-valued functional, defined on the ring $\mathcal{C}$ of closed convex sets in $\mathbb{R}^{N}$, such that $\chi(A)=0$ if $A=\varnothing, \chi(A)=1$ if $A$ is homotopic to the unit ball, and which satisfies the additivity property

$$
\chi(A \cup B)=\chi(A)+\chi(B)-\chi(A \cap B) \quad \text { for all } A, B \in \mathcal{C} .
$$

The investigation of its behaviour for the excursion sets of Gaussian random fields has now a rather long history: seminal contributions were given by Robert Adler and his coauthors in the 1970s; the area was then very much revived by the discovery of the beautiful Gaussian kinematic formula [1, 30].

Received April 2016; revised June 2017.

${ }^{1}$ Research supported by the ERC Grant n. 277742 Pascal (Probabilistic and Statistical Techniques for Cosmological Applications).

MSC2010 subject classifications. 60G60, 62M15, 53C65, 42C10, 33C55.

Key words and phrases. Euler-Poincaré characteristic, Wiener-chaos expansion, spherical harmonics, quantitative central limit theorem, Gaussian kinematic formula, Berry's cancellation phenomenon. 
More precisely, let us denote by $f$ a real valued random field defined on some manifold $\mathbb{M}$; as usual the excursion sets are defined by, for $u \in \mathbb{R}$,

$$
A_{u}(f ; \mathbb{M})=\{x \in \mathbb{M}: f(x) \geq u\} .
$$

We write $\mathcal{L}_{j}^{f}, j=0, \ldots, \operatorname{dim}(\mathbb{M})$, for the Lipschitz-Killing curvatures (also known as intrinsic volumes) of the manifold $\mathbb{M}$ under the Riemannian metric $g$ induced by the covariance of $f$; in other words, for $U_{x}, V_{x}$ that belong to $T_{x} \mathbb{M}$, the tangent space to $\mathbb{M}$ at $x$, we have

$$
g_{x}^{f}\left(U_{x}, V_{x}\right):=\mathbb{E}\left[\left(U_{x} f\right) \cdot\left(V_{x} f\right)\right]
$$

(see [1, 30] for further details); in particular $\mathcal{L}_{0}$ is the Euler-Poincaré characteristic. To introduce the Gaussian kinematic formula, we need to consider also the functions $\rho_{j}$, which are labelled Gaussian Minkowski functionals and defined by

$$
\rho_{j}(u)=(2 \pi)^{-(j+1) / 2} H_{j-1}(u) e^{-u^{2} / 2} ;
$$

here, $H_{q}(\cdot)$ are the Hermite polynomial of order $q$, which satisfy (see, e.g., [25])

$$
\begin{aligned}
H_{-1}(u) e^{-u^{2} / 2} & :=1-\Phi(u), \\
H_{j}(u) & =(-1)^{j}(\phi(u))^{-1} \frac{d^{j}}{d u^{j}} \phi(u), \quad j=0,1, \ldots
\end{aligned}
$$

$\phi(\cdot), \Phi(\cdot)$ denoting the standard Gaussian density and distribution functions, respectively. For instance, the first few Hermite polynomials are given by

$$
H_{0}(u)=1, \quad H_{1}(u)=u, \quad H_{2}(u)=u^{2}-1, \quad H_{3}(u)=u^{3}-3 u,
$$

For a smooth, centred, unit variance, Gaussian random field $f: \mathbb{M} \rightarrow \mathbb{R}$ the Gaussian kinematic formula then implies that the expected Euler-Poincaré characteristic of the excursion sets is given by

$$
\mathbb{E}\left[\chi\left(A_{u}(f ; \mathbb{M})\right)\right]=\sum_{j=0}^{\operatorname{dim}(\mathbb{M})} \mathcal{L}_{j}^{f}(\mathbb{M}) \rho_{j}(u) .
$$

More recently, a formula which can be viewed as an higher order extension of the Gaussian kinematic formula for the covariance of the Euler-Poincare characteristic of excursion sets at different thresholds, was established by [6], who focussed on an important class of fields: Gaussian spherical harmonics. Indeed, consider the Helmholtz equation

$$
\Delta_{\mathbb{S}^{2}} f_{\ell}+\lambda_{\ell} f_{\ell}=0, \quad f_{\ell}: \mathbb{S}^{2} \rightarrow \mathbb{R},
$$

where $\Delta_{\mathbb{S}^{2}}$ is the Laplace-Beltrami operator on the unit sphere $\mathbb{S}^{2}$ and $\lambda_{\ell}=\ell(\ell+$ $1), \ell=0,1,2, \ldots$. For a given eigenvalue $-\lambda_{\ell}$, the corresponding eigenspace is the $(2 \ell+1)$-dimensional space of spherical harmonics of degree $\ell$; we can choose 
an arbitrary $L^{2}$-orthonormal basis $\left\{Y_{\ell m}(\cdot)\right\}_{m=-\ell, \ldots, \ell}$, and consider random eigenfunctions of the form

$$
f_{\ell}(x)=\sqrt{\frac{4 \pi}{2 \ell+1}} \sum_{m=-\ell}^{\ell} a_{\ell m} Y_{\ell m}(x),
$$

where the coefficients $\left\{a_{\ell m}\right\}$ are complex-valued Gaussian variables, such that for $m \neq 0, \operatorname{Re}\left(a_{\ell m}\right), \operatorname{Im}\left(a_{\ell m}\right)$ are zero-mean, independent Gaussian variables with variance $\frac{1}{2}$, while $a_{\ell 0}$ follows a standard Gaussian distribution with zero mean and unit variance; the law of the process $\left\{f_{\ell}(\cdot)\right\}$ is invariant with respect to the choice of a $L^{2}$-orthonormal basis $\left\{Y_{\ell m}\right\}$. Note that in this paper we choose the basis of complex valued spherical harmonics instead of the real ones that were adopted in $[5,7]$. Random spherical harmonics arise naturally from Fourier analysis of isotropic spherical random fields and in the investigation of quantum chaos, and they have hence drawn quite a lot of interest in the last few years (see, for instance, [8, 19, 22, 24, 29, 32, 33]); as discussed below, we believe the results presented in this case can be extended to Gaussian eigenfunctions on more general compact manifolds, but we leave this issue for future research.

The random fields $\left\{f_{\ell}(x), x \in \mathbb{S}^{2}\right\}$ are centred, Gaussian and isotropic, meaning that the probability laws of $f_{\ell}(\cdot)$ and $f_{\ell}(g \cdot)$ are the same for any rotation $g \in$ $\mathrm{SO}(3)$. From the addition theorem for spherical harmonics ([17], equation (3.42)), the covariance function is given by

$$
\mathbb{E}\left[f_{\ell}(x) f_{\ell}(y)\right]=P_{\ell}(\cos d(x, y)),
$$

where $P_{\ell}$ are the Legendre polynomials and $d(x, y)$ is the spherical geodesic distance between $x$ and $y$, that is,

$$
d(x, y)=\arccos (\langle x, y\rangle)
$$

An application of the Gaussian kinematic formula (2) gives in these circumstances:

$$
\mathbb{E}\left[\chi\left(A_{u}\left(f_{\ell} ; \mathbb{S}^{2}\right)\right)\right]=\frac{\sqrt{2}}{\sqrt{\pi}} \exp \left\{-u^{2} / 2\right\} u \frac{\ell(\ell+1)}{2}+2[1-\Phi(u)],
$$

for a proof of formula (4); see, for example, [21], Corollary 5 or [10], Lemma 3.5. In [6], the results on the expected value were extended to an (asymptotic) evaluation of the variance; in particular, it was shown that, as $\ell \rightarrow \infty$

$$
\operatorname{Var}\left[\chi\left(A_{u}\left(f_{\ell} ; \mathbb{S}^{2}\right)\right)\right]=\frac{\ell^{3}}{8 \pi}\left(u^{3}-u\right)^{2} e^{-u^{2}}+O\left(\ell^{2} \log ^{2} \ell\right),
$$

an expression that can be rewritten as

$$
\ell \frac{\lambda \ell}{4}\left\{H_{1}(u) H_{2}(u) \phi(u)\right\}^{2}+O\left(\ell^{2} \log ^{2} \ell\right)
$$

or equivalently

$$
\ell \frac{\lambda \ell}{4}\left\{\left(H_{2}^{\prime}(u)+H_{3}(u)\right) \phi(u)\right\}^{2}+O\left(\ell^{2} \log ^{2} \ell\right),
$$


where $\phi(u)=\frac{1}{\sqrt{2 \pi}} e^{-u^{2} / 2}$ denotes as before the standard Gaussian density function. This expression was derived by an analytic computation, in turn a consequence of a rather hard analysis on the asymptotic variance of critical points which was given in [5, 7]. Asymptotic expressions for the variances of the two other Lipschitz-Killing curvatures for excursion sets in two dimensions, that is, the area and (half) the boundary length, were also given in [19, 22, 23] and [27, 33]; in [6] all these expressions were collected in a unitary framework and it was conjectured that they could point out to a more general formula for random eigenfunctions. A further contribution in this direction is indeed given by our results in this paper, which we present below.

1.1. Main results. The main purpose of this paper is to show that the high frequency behaviour is dominated (in the $L^{2}$ sense) by a single term with a very simple analytic expression, whose variance is indeed given by (6). In order to achieve this goal, we shall first establish the $L^{2}$ expansion of $\chi\left(A_{u}\left(f_{\ell} ; \mathbb{S}^{2}\right)\right)$ into Wiener chaoses [see (22) below], which we will write as

$$
\chi\left(A_{u}\left(f_{\ell} ; \mathbb{S}^{2}\right)\right)-\mathbb{E}\left[\chi\left(A_{u}\left(f_{\ell} ; \mathbb{S}^{2}\right)\right)\right]=\sum_{q=2}^{\infty} \operatorname{Proj}\left[\chi\left(A_{u}\left(f_{\ell} ; \mathbb{S}^{2}\right)\right) \mid q\right] .
$$

In the Euclidean case, a similar expansion was exploited in the recent paper [14]; in our setting, however, the asymptotic behaviour of the projection components turns out to be even neater; in particular, we shall show that the projection onto the second-order chaos has the following, very simple expression.

THEOREM 1. For all $\ell$ such that Condition 3 in Section 2.2 holds, we have

$$
\begin{aligned}
& \operatorname{Proj}\left[\chi\left(A_{u}\left(f_{\ell} ; \mathbb{S}^{2}\right)\right) \mid 2\right] \\
& =\frac{\lambda_{\ell}}{2}\left\{H_{1}(u) H_{2}(u) \phi(u)\right\} \frac{1}{2 \ell+1} \sum_{m=-\ell}^{\ell}\left\{\left|a_{\ell m}\right|^{2}-1\right\}+R(\ell) \\
& =\frac{\lambda_{\ell}}{2}\left\{H_{1}(u) H_{2}(u) \phi(u)\right\} \frac{1}{4 \pi} \int_{\mathbb{S}^{2}} H_{2}\left(f_{\ell}(x)\right) d x+R(\ell),
\end{aligned}
$$

where the remainder term $R(\ell)$ is such that $\mathbb{E}|R(\ell)|^{2}=O\left(\ell^{2} \log \ell\right)$, uniformly over $u$.

Note that the variance of the first term on the right-hand side is equal to

$$
\begin{aligned}
\operatorname{Var}[ & \left.\frac{\lambda \ell}{2}\left\{H_{1}(u) H_{2}(u) \phi(u)\right\} \frac{1}{2 \ell+1} \sum_{m=-\ell}^{\ell}\left\{\left|a_{\ell m}\right|^{2}-1\right\}\right] \\
& =\frac{\ell^{2}(\ell+1)^{2}}{4} \phi^{2}(u)\left(u^{3}-u\right)^{2} \frac{2}{2 \ell+1}=\frac{\ell^{3}}{8 \pi}\left(u^{3}-u\right)^{2} e^{-u^{2}}+O\left(\ell^{2}\right),
\end{aligned}
$$


which is asymptotically equivalent to the variance of the Euler-Poincaré characteristic reported in (5), so that the contribution from all the remaining Wiener chaos terms is indeed of smaller order for every $u \neq 0$. In view of this result, the investigation of the asymptotic distribution becomes indeed much less difficult, and we can prove the second main result of this paper, that is, we have the following.

THEOREM 2. There exists a constant $K>0$ such that, for all $\ell$ fulfilling Condition 3 in Section 2.2 and uniformly over $u \neq 0$, we have

$$
\mathbb{E}\left\{\frac{\chi\left(A_{u}\left(f_{\ell} ; \mathbb{S}^{2}\right)\right)-\mathbb{E}\left[\chi\left(A_{u}\left(f_{\ell} ; \mathbb{S}^{2}\right)\right)\right]-\operatorname{Proj}\left[\chi\left(A_{u}\left(f_{\ell} ; \mathbb{S}^{2}\right)\right) \mid 2\right]}{\sqrt{\operatorname{Var}\left[\chi\left(A_{u}\left(f_{\ell} ; \mathbb{S}^{2}\right)\right)\right]}}\right\}^{2} \leq K \frac{\log \ell}{\ell},
$$

and

$$
d_{W}\left(\frac{\chi\left(A_{u}\left(f_{\ell} ; \mathbb{S}^{2}\right)\right)-\mathbb{E}\left[\chi\left(A_{u}\left(f_{\ell} ; \mathbb{S}^{2}\right)\right)\right]}{\sqrt{\operatorname{Var}\left[\chi\left(A_{u}\left(f_{\ell} ; \mathbb{S}^{2}\right)\right)\right]}}, Z\right) \leq K \sqrt{\frac{\log \ell}{\ell}},
$$

$d_{W}(\cdot, \cdot)$ denoting as usual the Wasserstein distance and $Z \sim N(0,1)$ a standard Gaussian variable.

We remark that the possibility to obtain simple, analytic formulae for the second-order chaos component and its variance, together with sharp bounds on the convergence in Wasserstein distance, are both peculiar features which do not have analogous counterparts for the Euclidean domain results (see, e.g., [14]). Also, note that the asymptotic dependence on the threshold level $u$ is fully degenerate, that is, the Euler-Poincaré characteristic converges in mean square to a single random variable times a deterministic function of the threshold, in the high-frequency limit $\ell \rightarrow \infty$. All these features follow by the fact that a single chaotic projection (the component of order 2) is dominating the asymptotic behaviour of the EulerPoincare characteristic; this is in some sense similar to the reduction theorems that were established by M.Taqqu and coauthors for nonlinear functionals of long range dependent processes in a number of papers in the late 1970s and 1980s. For instance, in [13] it was proved that the asymptotic behaviour of the empirical process for long range dependent sequences is dominated by the second-order chaos components and, therefore, the limiting process boils down to a single random variable times a deterministic function of the parameter space. The analogy between the empirical process of long range dependent sequences and the excursion area for random eigenfunctions was first noted in [22]; here, we show that a similar behaviour holds for the Euler-Poincare characteristic, and indeed in the next subsection we discuss this issue in the more general framework of Lipschitz-Killing curvatures for excursion sets of Gaussian eigenfunctions. 


\subsection{Discussion.}

1.2.1. Some recent results on Lipschitz-Killing curvatures for Gaussian eigenfunctions. The fact that the asymptotic behaviour of the Lipschitz-Killing curvatures in the high frequency - high energy limit is dominated by the second-order chaotic component, which disappears at level $u=0$, seems to be of a general nature when dealing with excursion sets of random eigenfunctions. The simplest example of a Lipschitz-Killing curvature is given of course by the excursion area; in this case, it was shown in [22] that

$$
\begin{aligned}
\operatorname{Proj}\left[\mathcal{L}_{2}\left(A_{u}\left(f_{\ell} ; \mathbb{S}^{2}\right)\right) \mid 2\right] & =\frac{1}{2} u \phi(u) \int_{\mathbb{S}^{2}} H_{2}\left(f_{\ell}(x)\right) d x \\
& =\frac{1}{2} u \phi(u) \frac{4 \pi}{2 \ell+1} \sum_{m=-\ell}^{\ell}\left\{\left|a_{\ell m}\right|^{2}-1\right\}
\end{aligned}
$$

and moreover, as $\ell \rightarrow \infty$,

$$
\begin{aligned}
& \frac{\operatorname{Var}\left[\operatorname{Proj}\left[\mathcal{L}_{2}\left(A_{u}\left(f_{\ell} ; \mathbb{S}^{2}\right)\right) \mid 2\right]\right]}{\operatorname{Var}\left[\mathcal{L}_{2}\left(A_{u}\left(f_{\ell} ; \mathbb{S}^{2}\right)\right)\right]}=O\left(\frac{1}{\ell}\right), \\
& \lim _{\ell \rightarrow \infty} \mathbb{E}\left\{\frac{\mathcal{L}_{2}\left(A_{u}\left(f_{\ell} ; \mathbb{S}^{2}\right)\right)-\mathbb{E}\left[\mathcal{L}_{2}\left(A_{u}\left(f_{\ell} ; \mathbb{S}^{2}\right)\right)\right]-\operatorname{Proj}\left[\mathcal{L}_{2}\left(A_{u}\left(f_{\ell} ; \mathbb{S}^{2}\right)\right) \mid 2\right]}{\sqrt{\operatorname{Var}\left[\mathcal{L}_{2}\left(A_{u}\left(f_{\ell} ; \mathbb{S}^{2}\right)\right)\right]}}\right\}^{2}=0 .
\end{aligned}
$$

This results were further investigated and extended to spheres of arbitrary dimensions in [19]; again, easy consequences are:

1. A quantitative central limit theorem in Wasserstein distance;

2. Asymptotic degeneracy of the multivariate distribution for different thresholds $\left(u_{1}, \ldots, u_{p}\right)$, that is, perfect correlation of the excursion area at different thresholds;

3. The fact that the variance at level $u=0$ is lower-order (related to the socalled "Berry's cancellation phenomenon", see below).

Another step in this literature was the analysis of the boundary length for $u=0$ for random eigenfunctions on the torus, led by [18]; that is, the so-called nodal lines for arithmetic random waves, whose variance was first established in [15]. It should be noted that the nodal lines for arithmetic random waves are indeed (twice) their Lipschitz-Killing curvature of order 1 for $u=0$, that is, $\mathcal{L}_{1}\left(A_{0}\left(e_{k} ; \mathbb{T}^{2}\right)\right)$, where we use $\mathbb{T}^{2}$ to denote the two-dimensional torus and $e_{k}$ to denote its eigenfunctions, and $k$ is an integer such that $k^{2}=k_{1}^{2}+k_{2}^{2}$, for some $k_{1}, k_{2} \in \mathbb{N}$. The findings in [18] are indeed perfectly complementary to our investigation here: it is shown that the behaviour of nodal lines is dominated by a single term that corresponds to the fourth-order chaos component, consistent with the vanishing of the second-order term when $u=0$. Furthermore, in the (so far unpublished) Ph.D. 
thesis [27] it is shown that for the first Lipschitz-Killing curvature, that is, half the length of level curves of excursion sets of spherical eigenfunctions, one has also (Proposition 7.3.1, page 116)

$$
\begin{aligned}
\operatorname{Proj}\left[\mathcal{L}_{1}\left(A_{u}\left(f_{\ell} ; \mathbb{S}^{2}\right)\right) \mid 2\right] & =\frac{1}{2} \sqrt{\frac{\ell(\ell+1)}{2}} \sqrt{\frac{\pi}{8}} u^{2} \phi(u) \int_{\mathbb{S}^{2}} H_{2}\left(f_{\ell}(x)\right) d x \\
& =\frac{1}{2} \sqrt{\frac{\ell(\ell+1)}{2}} \sqrt{\frac{\pi}{8}} u^{2} \phi(u) \frac{4 \pi}{2 \ell+1} \sum_{m=-\ell}^{\ell}\left\{\left|a_{\ell m}\right|^{2}-1\right\},
\end{aligned}
$$

and thus again, as $\ell \rightarrow \infty$,

$$
\begin{aligned}
& \frac{\operatorname{Var}\left[\operatorname{Proj}\left[\mathcal{L}_{1}\left(A_{u}\left(f_{\ell} ; \mathbb{S}^{2}\right)\right) \mid 2\right]\right]}{\operatorname{Var}\left[\mathcal{L}_{1}\left(A_{u}\left(f_{\ell} ; \mathbb{S}^{2}\right)\right)\right]}=O\left(\frac{1}{\ell}\right), \\
& \lim _{\ell \rightarrow \infty} \mathbb{E}\left\{\frac{\mathcal{L}_{1}\left(A_{u}\left(f_{\ell} ; \mathbb{S}^{2}\right)\right)-\mathbb{E}\left[\mathcal{L}_{1}\left(A_{u}\left(f_{\ell} ; \mathbb{S}^{2}\right)\right)\right]-\operatorname{Proj}\left[\mathcal{L}_{1}\left(A_{u}\left(f_{\ell} ; \mathbb{S}^{2}\right)\right) \mid 2\right]}{\sqrt{\operatorname{Var}\left[\mathcal{L}_{1}\left(A_{u}\left(f_{\ell} ; \mathbb{S}^{2}\right)\right)\right]}}\right\}^{2}=0 .
\end{aligned}
$$

1.2.2. A possible unifying framework for spherical harmonics. The expressions we reported so far can be summarized into a single analytic form as follows, for $k=0,1,2$,

$$
\begin{aligned}
\operatorname{Proj}\left[\mathcal{L}_{k}\left(A_{u}\left(f_{\ell} ; \mathbb{S}^{2}\right)\right) \mid 2\right]= & \frac{1}{2}\left[\begin{array}{l}
2 \\
k
\end{array}\right]\left\{\frac{\lambda_{\ell}}{2}\right\}^{(2-k) / 2} H_{1}(u) H_{2-k}(u) \phi(u) \\
& \times \frac{1}{(2 \pi)^{(2-k) / 2}} \int_{\mathbb{S}^{2}} H_{2}\left(f_{\ell}(x)\right) d x+a_{k}(\ell),
\end{aligned}
$$

here, again we adopted the usual convention $H_{-1}(u) \phi(u):=1-\Phi(u)$; as in [1] we have introduced the flag coefficients:

$$
\left[\begin{array}{l}
2 \\
0
\end{array}\right]=\left[\begin{array}{l}
2 \\
2
\end{array}\right]=1, \quad\left[\begin{array}{l}
2 \\
1
\end{array}\right]=\frac{\pi}{2},
$$

and

$$
a_{k}(\ell)= \begin{cases}O_{p}(\ell) & \text { for } k=0, \\ 0 & \text { for } k=1,2 .\end{cases}
$$

It is important to notice that $\frac{\lambda_{\ell}}{2}=P_{\ell}^{\prime}(1)$ represents the derivative of the covariance function of random spherical harmonics at the origin, so that the term

$$
\frac{\lambda_{\ell}}{2} \int_{\mathbb{S}^{2}} H_{2}\left(f_{\ell}(x)\right) d x
$$

can be viewed as a (random) measure of the sphere induced by the Riemannian metric (1); recall indeed that for eigenfunctions $f_{\ell}$ on the sphere $\mathbb{S}^{2}$ the term 
$\mathcal{L}_{2}^{f_{\ell}}\left(\mathbb{S}^{2}\right)$ which appears in (2) is exactly given by the area of the sphere with radius $\left\{\frac{\lambda_{\ell}}{2}\right\}^{1 / 2}$, that is,

$$
\mathcal{L}_{2}^{f_{\ell}}\left(\mathbb{S}^{2}\right)=\frac{\lambda_{\ell}}{2} \times 4 \pi=\frac{\lambda_{\ell}}{2} \int_{\mathbb{S}^{2}} H_{0}\left(f_{\ell}(x)\right) d x
$$

At this stage, it seems very natural to notice that the expected value of LipschitzKilling curvatures can always be written as their projection on the Wiener chaos of order zero, that is, in our case

$$
\mathbb{E}\left[\mathcal{L}_{k}\left(A_{u}\left(f_{\ell} ; \mathbb{S}^{2}\right)\right)\right]=\operatorname{Proj}\left[\mathcal{L}_{k}\left(A_{u}\left(f_{\ell} ; \mathbb{S}^{2}\right)\right) \mid 0\right]
$$

so that we can rewrite the Gaussian kinematic formula with an expression which is remarkably similar to (7):

$$
\begin{aligned}
\operatorname{Proj}\left[\mathcal{L}_{k}\left(A_{u}\left(f_{\ell} ; \mathbb{S}^{2}\right)\right) \mid 0\right]= & {\left[\begin{array}{l}
2 \\
k
\end{array}\right]\left\{\frac{\lambda_{\ell}}{2}\right\}^{(2-k) / 2} H_{1-k}(u) \phi(u) } \\
& \times \frac{1}{(2 \pi)^{(2-k) / 2}} \int_{\mathbb{S}^{2}} H_{0}\left(f_{\ell}(x)\right) d x+b_{k}(\ell),
\end{aligned}
$$

where

$$
b_{k}(\ell)= \begin{cases}2(1-\Phi(u))=O(1) & \text { for } k=0 \\ 0 & \text { for } k=1,2 .\end{cases}
$$

The analogy between (7) and (8) is self-evident; more explicitly, combining the Gaussian kinematic formula with the results from [19, 22, 27] and those presented in this paper we have the following expressions for the projections $\operatorname{Proj}\left[\mathcal{L}_{k}\left(A_{u}\left(f_{\ell} ; \mathbb{S}^{2}\right)\right) \mid a\right], k=0,1,2, a=0,2$ :

(a) Excursion area $(k=2)$

$$
\begin{aligned}
& \operatorname{Proj}\left[\mathcal{L}_{2}\left(A_{u}\left(f_{\ell} ; \mathbb{S}^{2}\right)\right) \mid 0\right]=\left\{\frac{\lambda_{\ell}}{2}\right\}^{0}\left[H_{-1}(u) \phi(u)\right] \int_{\mathbb{S}^{2}} H_{0}\left(f_{\ell}(x)\right) d x, \\
& \operatorname{Proj}\left[\mathcal{L}_{2}\left(A_{u}\left(f_{\ell} ; \mathbb{S}^{2}\right)\right) \mid 2\right]=\frac{1}{2}\left\{\frac{\lambda_{\ell}}{2}\right\}^{0}\left[H_{0}(u) H_{1}(u) \phi(u)\right] \int_{\mathbb{S}^{2}} H_{2}\left(f_{\ell}(x)\right) d x
\end{aligned}
$$

(b) (Half) Boundary length $(k=1)$

(11) $\operatorname{Proj}\left[\mathcal{L}_{1}\left(A_{u}\left(f_{\ell} ; \mathbb{S}^{2}\right)\right) \mid 0\right]=\left\{\frac{\lambda_{\ell}}{2}\right\}^{1 / 2} \sqrt{\frac{\pi}{8}}\left[H_{0}(u) \phi(u)\right] \int_{\mathbb{S}^{2}} H_{0}\left(f_{\ell}(x)\right) d x$,

(12) $\operatorname{Proj}\left[\mathcal{L}_{1}\left(A_{u}\left(f_{\ell} ; \mathbb{S}^{2}\right)\right) \mid 2\right]=\frac{1}{2}\left\{\frac{\lambda_{\ell}}{2}\right\}^{1 / 2} \sqrt{\frac{\pi}{8}}\left[H_{1}^{2}(u) \phi(u)\right] \int_{\mathbb{S}^{2}} H_{2}\left(f_{\ell}(x)\right) d x$; 
(c) Euler-Poincaré characteristic $(k=0)$

$$
\begin{aligned}
\operatorname{Proj}\left[\mathcal{L}_{0}\left(A_{u}\left(f_{\ell} ; \mathbb{S}^{2}\right)\right) \mid 0\right]= & \left\{\frac{\lambda_{\ell}}{2}\right\}\left[H_{1}(u) \phi(u)\right] \frac{1}{2 \pi} \int_{\mathbb{S}^{2}} H_{0}\left(f_{\ell}(x)\right) d x \\
& +2\{1-\Phi(u)\}, \\
\operatorname{Proj}\left[\mathcal{L}_{0}\left(A_{u}\left(f_{\ell} ; \mathbb{S}^{2}\right)\right) \mid 2\right]= & \frac{1}{2}\left\{\frac{\lambda_{\ell}}{2}\right\}\left[H_{2}(u) H_{1}(u) \phi(u)\right] \frac{1}{2 \pi} \int_{\mathbb{S}^{2}} H_{2}\left(f_{\ell}(x)\right) d x \\
& +O_{p}(1) .
\end{aligned}
$$

1.3. Some comments and conjectures. We believe that the results we presented in this paper can shed some further light on a number of geometric features which have been noted in the literature on random spherical eigenfunctions. In particular, as noted earlier the asymptotic distribution for each of these Lipschitz-Killing curvatures is fully degenerate, as it is given by a single (standard Gaussian) random variable times a deterministic function of the threshold level $u$. Degeneracy of the limiting distribution provides an easy explanation for the full asymptotic correlation at different levels $u$ which was earlier noted for the Euler-Poincaré characteristic by [6]; for the length of level curves this phenomenon was observed in [33] and addressed in [27] (see also [18] for toral eigenfunctions), while for the excursion area asymptotic degeneracy was established by [22] and [19].

On the other hand, as noted already for the case of nodal lines by [18], the dominance of the second-order Wiener chaos and its disappearance for $u=0$ seems to provide a general explanation for the so-called Berry's cancellation phenomenon (see, e.g., [4, 32]), that is, the fact that the variance of these geometric functionals is of lower order in the ("nodal") case $u=0$ than for any other level $u \neq 0$. Indeed, the different asymptotic behaviour of these variances is due to the disappearance of the second-order Wiener chaos term; for the case of nodal length of arithmetic (toroidal) eigenfunctions, it was shown in [18] that the fourth-order chaos then dominates (see also [12, 26] for the complex-valued case and [20] for spherical nodal lines), while for the excursion area the case $u=0$ amounts to the so-called Defect, where all the odd-order chaotic components contribute in the limit (see [23]). Note that the second-order chaos components are proportional to the (random) fluctuations of the $L^{2}\left(\mathbb{S}^{2}\right)$ norm of the eigenfunctions, that is,

$$
\begin{aligned}
\int_{\mathbb{S}^{2}} H_{2}\left(f_{\ell}(x)\right) d x & =\int_{\mathbb{S}^{2}}\left\{f_{\ell}^{2}(x)-1\right\} d x=\int_{\mathbb{S}^{2}} f_{\ell}^{2}(x) d x-4 \pi \\
& =\left\|f_{\ell}\right\|_{L^{2}\left(\mathbb{S}^{2}\right)}^{2}-\mathbb{E}\left\|f_{\ell}\right\|_{L^{2}\left(\mathbb{S}^{2}\right)}^{2} ;
\end{aligned}
$$

the fact that second-order chaos components $(10,12,14)$ vanish in the nodal case $u=0$ is thus in some sense rather natural, because the excursion regions at the zero level are clearly unaffected by (multiplicative) scaling factors, and hence independent from the $L^{2}\left(\mathbb{S}^{2}\right)$ norm. 
Dealing with eigenfunctions does entail some special relationships between the field and its second-order derivatives, hence we do not expect the same results to hold for general classes of isotropic Gaussian fields. On the one hand, we do expect these phenomena to hold in greater generality than discussed here; in particular, we conjecture that for random eigenfunctions on compact manifolds with increasing spectral multiplicities the asymptotic behaviour of Lipschitz-Killing curvatures of excursion sets at any level $u \neq 0$ is dominated, in the high-energy limit, by the projection on the second-order Wiener chaos; we also expect this leading component to vanish in the nodal case $u=0$, hence yielding a phase transition to lower order variance behaviour. Among the compact manifolds with eigenfunctions which exhibit spectral degeneracies (i.e., eigenspaces of dimensions larger than one) there are, of course the sphere $\mathbb{S}^{d}$ and the torus $\mathbb{T}^{d}$ in arbitrary dimensions $d \geq 2$; a future challenge for research is the derivation of general expressions akin to (7) for the behaviour of Lipschitz-Killing curvatures in these more general settings. How to cover these significantly wider classes of covariances and/or manifolds is however still unclear at this moment.

1.4. Plan of the paper. The plan of this paper is as follows: in Section 2 we review some background material and our notation; Section 3 discusses the projection of the Euler-Poincaré characteristic into second-order chaos, while Section 4 collects the exact computation of the variance and the proof of the quantitative central limit theorem. A number of technical and auxiliary results are collected in Section 5.

\section{Background and notation.}

2.1. Morse theory. As it is customary in this branch of literature, we shall exploit a general representation for the Euler-Poincaré characteristic in terms of critical points by means of so-called Morse theory (see [1], Section 9.3). Indeed, assuming that $\mathbb{M}$ is a $C^{2}$ manifold without boundary in $\mathbb{R}^{N}$ and that $h \in C^{2}(\mathbb{M})$ is a Morse function on $\mathbb{M}$ (i.e., its Hessian is nondegenerate at the critical points), it is well known that the Euler-Poincaré characteristic can be expressed as an alternating sum:

$$
\chi(\mathbb{M})=\sum_{j=0}^{\operatorname{dim}(\mathbb{M})}(-1)^{j} \mu_{j}(\mathbb{M}, h),
$$

where $\mu_{j}(\mathbb{M}, h)$ is the number of critical points of $h$ with Morse index $j$, that is, the Hessian of $h$ has $j$ negative eigenvalues; for a proof of (15), see [1], Corollary 9.3.3. To establish our results, we will make use of (15) in the case of excursion sets of spherical eigenfunctions; to this aim, we recall some basic differential 
geometry on $\mathbb{S}^{2}$, along the same lines as we did in [6]. More precisely, let us recall that the metric tensor on the tangent plane $T\left(\mathbb{S}^{2}\right)$ is given by

$$
g(\theta, \varphi)=\left[\begin{array}{cc}
1 & 0 \\
0 & \sin ^{2} \theta
\end{array}\right]
$$

For $x=(\theta, \varphi) \in \mathbb{S}^{2} \backslash\{N, S\}(N, S$ are the north and south poles, that is, $\theta=0$ and $\theta=\pi$, resp.), the vectors

$$
e_{1}^{x}=\vec{e}_{\theta}=\frac{\partial}{\partial \theta}, \quad e_{2}^{x}=\vec{e}_{\varphi}=\frac{1}{\sin \theta} \frac{\partial}{\partial \varphi},
$$

constitute an orthonormal basis for $T_{x}\left(\mathbb{S}^{2}\right)$; in these system of coordinates the gradient is given by $\nabla=\left(\frac{\partial}{\partial \theta}, \frac{1}{\sin \theta} \frac{\partial}{\partial \varphi}\right)$. As usual, the Hessian of a function $f \in C^{2}\left(\mathbb{S}^{2}\right)$ is defined as the bilinear symmetric map from $C^{1}\left(T\left(\mathbb{S}^{2}\right)\right) \times C^{1}\left(T\left(\mathbb{S}^{2}\right)\right)$ to $C^{0}\left(\mathbb{S}^{2}\right)$ given by

$$
\nabla_{E}^{2} f(X, Y)=X Y f-\nabla_{X} Y f, \quad X, Y \in T\left(\mathbb{S}^{2}\right),
$$

where $\nabla_{X}$ denotes Levi-Civita connection (see, e.g., [1], Chapter 7 for more discussion and details). For our computations to follow, we shall need the matrixvalued process $\nabla_{E}^{2} f_{\ell}(x)$ with elements given by

$$
\left\{\nabla_{E}^{2} f_{\ell}(x)\right\}_{a, b=\theta, \varphi}=\left\{\left(\nabla^{2} f_{\ell}(x)\right)\left(\vec{e}_{a}, \vec{e}_{b}\right)\right\}_{a, b=\theta, \varphi},
$$

where $E=\left\{\vec{e}_{\theta}, \vec{e}_{\varphi}\right\}$. With the standard system of spherical coordinates, the analytic expression for this matrix is given by

$\nabla_{E}^{2} f_{\ell}(x)$

$$
\begin{aligned}
& =\left[\begin{array}{cc}
\frac{\partial^{2}}{\partial \theta^{2}}-\Gamma_{\theta \theta}^{\theta} \frac{\partial}{\partial \theta}-\Gamma_{\theta \theta}^{\varphi} \frac{\partial}{\partial \varphi} & \frac{1}{\sin \theta}\left[\frac{\partial^{2}}{\partial \theta \partial \varphi}-\Gamma_{\varphi \theta}^{\varphi} \frac{\partial}{\partial \varphi}-\Gamma_{\theta \varphi}^{\theta} \frac{\partial}{\partial \theta}\right] \\
\frac{1}{\sin \theta}\left[\frac{\partial^{2}}{\partial \theta \partial \varphi}-\Gamma_{\varphi \theta}^{\varphi} \frac{\partial}{\partial \varphi}-\Gamma_{\theta \varphi}^{\theta} \frac{\partial}{\partial \theta}\right] & \frac{1}{\sin ^{2} \theta}\left[\frac{\partial^{2}}{\partial \varphi^{2}}-\Gamma_{\varphi \varphi}^{\varphi} \frac{\partial}{\partial \varphi}-\Gamma_{\varphi \varphi}^{\theta} \frac{\partial}{\partial \theta}\right]
\end{array}\right] \\
& =\left[\begin{array}{cc}
\frac{\partial^{2}}{\partial \theta^{2}} & \frac{1}{\sin \theta}\left[\frac{\partial^{2}}{\partial \theta \partial \varphi}-\frac{\cos \theta}{\sin \theta} \frac{\partial}{\partial \varphi}\right] \\
\frac{1}{\sin \theta}\left[\frac{\partial^{2}}{\partial \theta \partial \varphi}-\frac{\cos \theta}{\sin \theta} \frac{\partial}{\partial \varphi}\right] & \frac{1}{\sin ^{2} \theta}\left[\frac{\partial^{2}}{\partial \varphi^{2}}+\sin \theta \cos \theta \frac{\partial}{\partial \theta}\right]
\end{array}\right],
\end{aligned}
$$

where $\Gamma_{a b}^{c}$ are the usual Christoffel symbols (see, e.g., [9] Section I.1), from which we can compute the Levi-Civita connection:

$$
\nabla_{\vec{e}_{a}} \vec{e}_{b}=\Gamma_{a b}^{\theta} \vec{e}_{\theta}+\Gamma_{a b}^{\varphi} \vec{e}_{\varphi}, \quad a, b=\theta, \varphi .
$$

More explicitly, Christoffel symbols for $\mathbb{S}^{2}$ are given by

$$
\Gamma_{\theta \varphi}^{\theta}=\Gamma_{\theta \theta}^{\theta}=\Gamma_{\varphi \varphi}^{\varphi}=\Gamma_{\theta \theta}^{\varphi}=0, \quad \Gamma_{\varphi \varphi}^{\theta}=-\sin \theta \cos \theta, \quad \Gamma_{\varphi \theta}^{\varphi}=\cot \theta .
$$


For every $x \in \mathbb{S}^{2}$, let $\nabla f_{\ell}(x)$ and $\nabla^{2} f_{\ell}(x)$ be the vector-valued processes with elements

$$
\begin{aligned}
\nabla f_{\ell}(x) & =\left(e_{1}^{x} f_{\ell}(x), e_{2}^{x} f_{\ell}(x)\right), \\
\nabla^{2} f_{\ell}(x) & =\left(e_{1}^{x} e_{1}^{x} f_{\ell}(x), e_{1}^{x} e_{2}^{x} f_{\ell}(x), e_{2}^{x} e_{2}^{x} f_{\ell}(x)\right) .
\end{aligned}
$$

Since the $f_{\ell}$ are eigenfunctions of the spherical Laplacian, the value of $f_{\ell}$ at every fixed point $x \in \mathbb{S}^{2}$ is a linear combination of its first- and second-order derivatives at $x$. If the point $x \in \mathbb{S}^{2}$ is also a critical point for $f_{\ell}$, it follows that the value of the spherical harmonic at $x$ is a linear combination of its second-order derivatives, that is,

$$
e_{1}^{x} e_{1}^{x} f_{\ell}(x)+e_{2}^{x} e_{2}^{x} f_{\ell}(x)=-\lambda_{\ell} f_{\ell}(x) .
$$

Let us take $\mathbb{M}$ and $h$ in formula $(15)$ to be $A_{u}\left(f_{\ell} ; \mathbb{S}^{2}\right)$ and $\left.f_{\ell}\right|_{A_{u}\left(f_{\ell} ; \mathbb{S}^{2}\right)}$, respectively; by the Morse representation, we obtain

$$
\chi\left(A_{u}\left(f_{\ell} ; \mathbb{S}^{2}\right)\right)=\sum_{j=0}^{2}(-1)^{j} \mu_{j},
$$

where

$$
\begin{aligned}
\mu_{j}= & \#\left\{x \in \mathbb{S}^{2}: f_{\ell}(x) \geq u, \nabla f_{\ell}(x)=0, \operatorname{Ind}\left(-\nabla^{2} f_{\ell}(x)\right)=j\right\} \\
= & \#\left\{x \in \mathbb{S}^{2}: e_{1}^{x} e_{1}^{x} f_{\ell}(x)+e_{2}^{x} e_{2}^{x} f_{\ell}(x) \leq-\lambda_{\ell} u,\right. \\
& \left.\nabla f_{\ell}(x)=0, \operatorname{Ind}\left(-\nabla^{2} f_{\ell}(x)\right)=j\right\}
\end{aligned}
$$

Ind $(M)$ denoting the number of negative eigenvalues of a square matrix $M$. More specifically, $\mu_{0}$ is the number of maxima, $\mu_{1}$ the number of saddles, and $\mu_{2}$ the number of minima in the excursion region $A_{u}\left(f_{\ell} ; \mathbb{S}^{2}\right)$. In the next subsection, we show how to justify this representation into a $L^{2}$ space, by means of an approximating sequence of delta functions.

2.2. The delta function approximation. Let us now denote by $\Sigma_{\ell}(x, y)$ the covariance matrix for the 10-dimensional Gaussian random vector

$$
\left(\nabla f_{\ell}(x), \nabla f_{\ell}(y), \nabla^{2} f_{\ell}(x), \nabla^{2} f_{\ell}(y)\right),
$$

which combines the gradient and the elements of the Hessian evaluated at $x, y$; we shall write

$$
\Sigma_{\ell}(x, y)=\left(\begin{array}{ll}
A_{\ell}(x, y) & B_{\ell}(x, y) \\
B_{\ell}^{t}(x, y) & C_{\ell}(x, y)
\end{array}\right)
$$

where the $A_{\ell}$ and $C_{\ell}$ components collect the variances of the gradient and Hessian terms, respectively, while the matrix $B_{\ell}$ collects the covariances between first- and second-order derivatives. The explicit computation of $\Sigma_{\ell}(x, y)$ requires iterative derivations of Legendre polynomials and are given in [5], Appendix 1. For the $L^{2}$ expansion of the Euler-Poincaré characteristic to hold, we need to assume the following, standard nondegeneracy condition. 
CONDITION 3. For every $(x, y) \in \mathbb{S}^{2}$, the Gaussian vector $\left(\nabla f_{\ell}(x), \nabla f_{\ell}(y)\right)$ has a nondegenerate density function, that is, the covariance matrix $A_{\ell}(x, y)$ is invertible.

We can now build an approximating sequence of delta functions, and establish their convergence both in the a.s. and in the $L^{2}$ sense. More precisely, let $\delta_{\varepsilon}: \mathbb{R}^{2} \rightarrow$ $\mathbb{R}$ be such that

$$
\delta_{\varepsilon}(z)=(2 \varepsilon)^{-2} \mathbb{I}_{[-\varepsilon, \varepsilon]^{2}}(z),
$$

and define the approximating sequence

$$
\chi_{\varepsilon}\left(A_{u}\left(f_{\ell} ; \mathbb{S}^{2}\right)\right)=\sum_{j=0}^{2} \mu_{j}(\varepsilon)
$$

where

$$
\mu_{j}(\varepsilon)=\int_{\mathbb{S}^{2}}\left|\operatorname{det}\left(\nabla^{2} f_{\ell}(x)\right)\right| \mathbb{I}_{\left\{\tilde{f}_{\ell}(x) \geq u\right\}} \mathbb{I}_{\left\{\operatorname{Ind}\left(-\nabla^{2} f_{\ell}(x)\right)=j\right\}} \delta_{\varepsilon}\left(\nabla f_{\ell}(x)\right) d x
$$

and we wrote for brevity

$$
\tilde{f}_{\ell}(x):=-\frac{e_{1}^{x} e_{1}^{x} f_{\ell}(x)+e_{2}^{x} e_{2}^{x} f_{\ell}(x)}{\lambda_{\ell}} ;
$$

note that $\tilde{f}_{\ell}(x)=f_{\ell}(x)$ when $x$ is a critical point, that is, as $\varepsilon \rightarrow 0$. Now recall the standard identity (see, e.g., [2], Lemma 4.2.2)

$$
\sum_{j=0}^{2}(-1)^{j}\left|\operatorname{det}\left(\nabla^{2} f_{\ell}(x)\right)\right| \mathbb{I}_{\left\{\operatorname{Ind}\left(-\nabla^{2} f_{\ell}(x)\right)=j\right\}}=\operatorname{det}\left(-\nabla^{2} f_{\ell}(x)\right)
$$

so that we can rewrite $\chi_{\varepsilon}\left(A_{u}\left(f_{\ell} ; \mathbb{S}^{2}\right)\right)$ as

$$
\chi_{\varepsilon}\left(A_{u}\left(f_{\ell} ; \mathbb{S}^{2}\right)\right)=\int_{\mathbb{S}^{2}} \operatorname{det}\left(\nabla^{2} f_{\ell}(x)\right) \mathbb{I}_{\left\{\tilde{f}_{\ell}(x) \geq u\right\}} \delta_{\varepsilon}\left(\nabla f_{\ell}(x)\right) d x .
$$

We are now able to prove the almost sure and $L^{2}(\Omega)$ convergence of $\chi_{\varepsilon}\left(A_{u}\left(f_{\ell}\right.\right.$; $\left.\mathbb{S}^{2}\right)$ ) to $\chi\left(A_{u}\left(f_{\ell} ; \mathbb{S}^{2}\right)\right)$, as $\varepsilon \rightarrow 0$; this result has been already shown to hold for a very general class of Gaussian fields in Euclidean spaces; see, for example, [14].

LEMMA 4. For every $\ell$ such that Condition 3 holds, we have

$$
\chi\left(A_{u}\left(f_{\ell} ; \mathbb{S}^{2}\right)\right)=\lim _{\varepsilon \rightarrow 0} \chi_{\varepsilon}\left(A_{u}\left(f_{\ell} ; \mathbb{S}^{2}\right)\right),
$$

where the convergence holds both $\omega$-a.s. and in $L^{2}(\Omega)$. 
PROOF. To prove almost sure convergence, we first apply [1], Theorem 11.2.3, where we take $f=\nabla f_{\ell}: \mathbb{S}^{2} \rightarrow \mathbb{R}^{2}, g=\left(f_{\ell},-\nabla^{2} f_{\ell}\right): \mathbb{S}^{2} \rightarrow \mathbb{R}^{4}, u=0$ and $B=$ $B_{j}=[u, \infty) \times\{$ Ind $=j\}$, so that, for $j=0,1,2$, we have

$$
\mu_{j}=\lim _{\varepsilon \rightarrow 0} \mu_{j}(\varepsilon), \quad \omega \text {-a.s. }
$$

We note that the conditions in [1], Theorem 11.2.3, are all fulfilled since random spherical harmonics are Morse functions with probability one, under Condition 3; then the almost sure convergence (19) immediately follows from (17), (20) and (18). We prove now that (19) also holds in $L^{2}(\Omega)$; it is a classical result that $L^{2}$-convergence follows from convergence a.s. and convergence of the $L^{2}$ norm, whence the proof will be completed if we show that

$$
\lim _{\varepsilon \rightarrow 0} \mathbb{E}\left[\chi_{\varepsilon}\left(A_{u}\left(f_{\ell} ; \mathbb{S}^{2}\right)\right)\right]^{2}=\mathbb{E}\left[\chi\left(A_{u}\left(f_{\ell} ; \mathbb{S}^{2}\right)\right)\right]^{2}
$$

Indeed, note that

$$
\mathbb{E}\left[\chi_{\varepsilon}\left(A_{u}\left(f_{\ell} ; \mathbb{S}^{2}\right)\right)\right]^{2}=\sum_{j, k=0}^{2}(-1)^{j+k} \mathbb{E}\left[\mu_{j}(\varepsilon) \mu_{k}(\varepsilon)\right] .
$$

Under Condition 3, we can apply Kac-Rice formula to compute $\mathbb{E}\left[\mu_{j}(\varepsilon) \mu_{k}(\varepsilon)\right]$ (see [3], Theorem 6.3 or [1], Theorem 11.2.1) and, proceeding as in the proof of [6], Proposition 1, we obtain

$$
\sum_{j, k=0}^{2}(-1)^{j+k} \mathbb{E}\left[\mu_{j}(\varepsilon) \mu_{k}(\varepsilon)\right]=\int_{\mathbb{S}^{2}} \int_{\mathbb{S}^{2}} \int_{u}^{\infty} \int_{u}^{\infty} J_{2, \ell, \varepsilon}\left(x, y, t_{1}, t_{2}\right) d t_{1} d t_{2} d x d y,
$$

where

$$
\begin{aligned}
J_{2, \ell, \varepsilon}\left(x, y, t_{1}, t_{2}\right) \\
=\frac{1}{(2 \varepsilon)^{4}} \iint_{[-\varepsilon, \varepsilon]^{2} \times[-\varepsilon, \varepsilon]^{2}} d \eta_{1} d \eta_{2}\left\{\varphi_{\left(\tilde{f}_{\ell}(x), \tilde{f}_{\ell}(y), \nabla f_{\ell}(x), \nabla f_{\ell}(y)\right)}\left(t_{1}, t_{2}, \eta_{1}, \eta_{2}\right)\right. \\
\quad \times \mathbb{E}\left[\operatorname{det}\left(-\nabla^{2} f_{\ell}(x)\right) \operatorname{det}\left(-\nabla^{2} f_{\ell}(y)\right) \mid \nabla f_{\ell}(x)=\eta_{1}, \nabla f_{\ell}(y)=\eta_{2},\right. \\
\left.\left.\quad \tilde{f}_{\ell}(x)=t_{1}, \tilde{f}_{\ell}(y)=t_{2}\right]\right\},
\end{aligned}
$$

and $\varphi_{\left(\tilde{f}_{\ell}(x), \tilde{f}_{\ell}(y), \nabla f_{\ell}(x), \nabla f_{\ell}(y)\right)}$ is the density of the 6-dimensional vector

$$
\left(\tilde{f}_{\ell}(x), \tilde{f}_{\ell}(y), \nabla f_{\ell}(x), \nabla f_{\ell}(y)\right) .
$$

We note also that, under Condition 3 , the covariance matrix $A_{\ell}(x, y)$ and the conditional covariance matrix of the Gaussian vector

$$
\left(\nabla^{2} f_{\ell}(x), \nabla^{2} f_{\ell}(y) \mid \nabla f_{\ell}(x), \nabla f_{\ell}(y), \tilde{f}_{\ell}(x), \tilde{f}_{\ell}(y)\right)
$$


are invertible for $x, y \in \mathbb{S}^{2}$; hence the conditional Gaussian density function is continuous, and thus, as $\varepsilon \rightarrow 0$, the integral $J_{2, \ell, \varepsilon}\left(x, y, t_{1}, t_{2}\right)$ converges to

$$
\begin{aligned}
J_{2, \ell}(x, & \left.y, t_{1}, t_{2}\right) \\
= & \varphi_{\left(f_{\ell}(x), f_{\ell}(y), \nabla f_{\ell}(x), \nabla f_{\ell}(y)\right)}\left(t_{1}, t_{2}, 0,0\right) \\
& \times \mathbb{E}\left[\operatorname{det}\left(-\nabla^{2} f_{\ell}(x)\right) \operatorname{det}\left(-\nabla^{2} f_{\ell}(y)\right) \mid f_{\ell}(x)=t_{1}, f_{\ell}(y)=t_{2},\right. \\
& \left.\nabla f_{\ell}(x)=0, \nabla f_{\ell}(y)=0\right] .
\end{aligned}
$$

The statement follows by observing that under Condition 3, and in view of [6], Proposition 1, we also have

$$
\mathbb{E}\left[\chi\left(A_{u}\left(f_{\ell} ; \mathbb{S}^{2}\right)\right)\right]^{2}=\int_{\mathbb{S}^{2}} \int_{\mathbb{S}^{2}} \int_{u}^{\infty} \int_{u}^{\infty} J_{2, \ell}\left(x, y, t_{1}, t_{2}\right) d t_{1} d t_{2} d x d y .
$$

2.3. Wiener chaos. In this section, we recall very briefly some basic facts on Wiener-Itô chaotic expansion for nonlinear functionals of Gaussian fields. We follow closely the summary which was given in [18], while we refer to [25] for an exhaustive discussion.

Recall first that each random eigenfunction $f_{\ell}$ in (3) is a by-product of the family of complex-valued, independent, Gaussian random variables $\left\{a_{\ell m}\right\}, m=$ $-\ell, \ldots, \ell$, defined on some probability space $(\Omega, \mathcal{F}, \mathbb{P})$ and satisfying the following properties: (i) for $m \neq 0$ every $a_{\ell m}$ has the form

$$
\operatorname{Re}\left(a_{\ell m}\right)+i \operatorname{Im}\left(a_{\ell m}\right),
$$

where $\operatorname{Re}\left(a_{\ell m}\right)$ and $\operatorname{Im}\left(a_{\ell m}\right)$ are two zero-mean, independent Gaussian variables with variance $1 / 2$; (ii) $a_{\ell 0}$ follows a standard Gaussian distribution; (iii) $a_{\ell, m}$ and $a_{\ell, m^{\prime}}$ are stochastically independent whenever $m^{\prime} \neq-m$; (iv) $(-1)^{m} a_{\ell,-m}=\bar{a}_{\ell m}$. We define the space $\mathbf{A}$ to be the closure in $L^{2}(\mathbb{P})$ of all real finite linear combinations of random variables of the forms

$$
z(-1)^{m} a_{\ell,-m}+\bar{z} a_{\ell m} \text { and } a_{\ell 0},
$$

$z \in \mathbb{C}$; the space $A$ is a real, centred, Gaussian Hilbert subspace of $L^{2}(\mathbb{P})$. For each $q \geq 0$, the $q$ th Wiener chaos $\mathcal{H}_{q}$ associated with $\mathbf{A}$ is the closed linear subspace of $L^{2}(\mathbb{P})$ generated by all real, finite, linear combinations of random variables of the form

$$
H_{q_{1}}\left(x_{1}\right) \cdot H_{q_{2}}\left(x_{2}\right) \cdots H_{q_{k}}\left(x_{k}\right)
$$

for $k \geq 1$, where the integers $q_{1}, q_{2}, \ldots, q_{k} \geq 0$ satisfy $q_{1}+q_{2}+\cdots+q_{k}=q$, and $\left(x_{1}, x_{2}, \ldots, x_{k}\right)$ is a standard, real, Gaussian vector extracted form $\mathbf{A}$; note that in particular $\mathcal{H}_{0}=\mathbb{R}$. As well-known Wiener chaoses $\left\{\mathcal{H}_{q}, q=0,1,2, \ldots\right\}$ are orthogonal, that is, $\mathcal{H}_{q} \perp \mathcal{H}_{p}$ for $p \neq q$; moreover, the following Wiener-Ito 
decomposition of $L^{2}(\mathbb{P})$ holds: every random variable $F \in L^{2}(\mathbb{P})$ admits a unique expansion of the type

$$
F=\mathbb{E}[F]+\sum_{q=1}^{\infty} \operatorname{Proj}[F \mid q]
$$

where the projections $\operatorname{Proj}[F \mid q] \in \mathcal{H}_{q}$ for every $q=1,2, \ldots$ and the series converges in $L^{2}(\mathbb{P})$. Again we refer [25], Theorem 2.2.4, for an extremely rich discussion and a vast gallery of examples and applications.

2.4. Overview of the proof. The main technical tools for our argument are collected in Proposition 5 and Proposition 6; the proof of each of these results takes a separate subsection in Section 5. In particular, in Proposition 5 we derive explicit analytic expression for the projection coefficients on the components of secondorder Wiener chaos; in Proposition 6, we manage to write down the integrals over the sphere of these components in terms of weighted sums of the random spherical harmonic coefficients $\left\{a_{\ell m}\right\}$ : the latter results requires a very careful analytic investigation on derivatives of associated Legendre function, which is given in Section 5. Combining together Proposition 5 and Proposition 6, one obtains an explicit formula for the second-order Wiener chaos, which can be further simplified by some algebraic manipulations to achieve the statement of Theorem 1. Because the spherical harmonic coefficients are independent and identically distributed (excluding the term at $m=0$ ), the conclusions of Theorem 2 are then rather straightforward to obtain.

3. The projection into the second Wiener chaos. In this section, we prove Theorem 1, that is, we derive an analytic expression for the projection of the EulerPoincaré characteristic on the second-order Wiener chaos. Our strategy for this proof can be summarized as follows: from standard results in Morse theory detailed in the previous section, we can express the Euler-Poincaré characteristic as a function of a six-dimensional vector, involving the eigenfunctions $f_{\ell}$, the two-dimensional gradient vector and the three-dimensional vector including the independent components of the Hessian. Actually, as in [5] these components may immediately be reduced to five, as the eigenfunctions can be written as linear combinations of first- and second-order derivatives. It is then convenient to implement a linear transform on this vector, to make its components independent when evaluated on the same point $x \in \mathbb{S}^{2}$; this idea is analogous to the approach which was pursue by [14] in their recent work on the Euler-Poincare characteristic for Gaussian field on an Euclidean (growing) domain. We are then able to write down explicitly the projection coefficients on the second-order Wiener chaos; the result then follows from a very careful cancellation of the different projection components. 
3.1. Cholesky decomposition. In view of (16), it follows that we can rewrite $\chi_{\varepsilon}\left(A_{u}\left(f_{\ell} ; \mathbb{S}^{2}\right)\right)$ as

$$
\begin{aligned}
\chi_{\varepsilon}\left(A_{u}\left(f_{\ell} ; \mathbb{S}^{2}\right)\right) \\
=\int_{\mathbb{S}^{2}}\left[e_{1}^{x} e_{1}^{x} f_{\ell}(x) e_{2}^{x} e_{2}^{x} f_{\ell}(x)-\left(e_{1}^{x} e_{2}^{x} f_{\ell}(x)\right)^{2}\right] \mathbb{I}_{\left\{e_{1}^{x} e_{1}^{x} f_{\ell}(x)+e_{2}^{x} e_{2}^{x} f_{\ell}(x) \leq-\lambda_{\ell} u\right\}} \\
\quad \times \delta_{\varepsilon}\left(e_{1}^{x} f_{\ell}(x), e_{2}^{x} f_{\ell}(x)\right) d x .
\end{aligned}
$$

It should be noted that the integrand

$$
\begin{aligned}
& {\left[e_{1}^{x} e_{1}^{x} f_{\ell}(x) e_{2}^{x} e_{2}^{x} f_{\ell}(x)-\left(e_{1}^{x} e_{2}^{x} f_{\ell}(x)\right)^{2}\right]} \\
& \quad \times \mathbb{I}_{\left\{e_{1}^{x} e_{1}^{x} f_{\ell}(x)+e_{2}^{x} e_{2}^{x} f_{\ell}(x) \leq-\lambda_{\ell} u\right\}} \delta_{\varepsilon}\left(e_{1}^{x} f_{\ell}(x), e_{2}^{x} f_{\ell}(x)\right)
\end{aligned}
$$

is isotropic, so focussing on the great circle $\theta_{x}=\frac{\pi}{2}$ is simply a convenient simplification. Let us now write $\sigma_{\ell}(x)$ for the $5 \times 5$ covariance matrix of the Gaussian random vector

$$
\left(e_{1}^{x} f_{\ell}(x), e_{2}^{x} f_{\ell}(x), e_{1}^{x} e_{1}^{x} f_{\ell}(x), e_{1}^{x} e_{2}^{x} f_{\ell}(x), e_{2}^{x} e_{2}^{x} f_{\ell}(x)\right),
$$

that is, the $5 \times 1$ vector that includes the gradient and the Hessian components of interest. We evaluate the covariance matrix $\sigma_{\ell}(x)$ on the great circle such that $\theta_{x}=\frac{\pi}{2}$, and we write it in the partitioned form

$$
\sigma_{\ell}(x)_{5 \times 5}=\left(\begin{array}{ll}
a_{\ell}(x) & b_{\ell}(x) \\
b_{\ell}^{t}(x) & c_{\ell}(x)
\end{array}\right),
$$

where the superscript $t$ denotes transposition, and (see [5], Section 2.2)

$$
\begin{aligned}
& a_{\ell}\left(\frac{\pi}{2}, \varphi\right)=\left(\begin{array}{cc}
\frac{\lambda_{\ell}}{2} & 0 \\
0 & \frac{\lambda_{\ell}}{2}
\end{array}\right), \quad b_{\ell}\left(\frac{\pi}{2}, \varphi\right)=\left(\begin{array}{lll}
0 & 0 & 0 \\
0 & 0 & 0
\end{array}\right), \\
& c_{\ell}\left(\frac{\pi}{2}, \varphi\right)=\frac{\lambda_{\ell}^{2}}{8}\left(\begin{array}{ccc}
3-\frac{2}{\lambda_{\ell}} & 0 & 1+\frac{2}{\lambda_{\ell}} \\
0 & 1-\frac{2}{\lambda_{\ell}} & 0 \\
1+\frac{2}{\lambda_{\ell}} & 0 & 3-\frac{2}{\lambda_{\ell}}
\end{array}\right) .
\end{aligned}
$$

Let us first recall that the Cholesky decomposition of a Hermitian positive-definite matrix $A$ takes the form $A=\Lambda \Lambda^{t}$, where $\Lambda$ is a lower triangular matrix with real and positive diagonal entries, and $\Lambda^{t}$ denotes the conjugate transpose of $\Lambda$. It is well known that every Hermitian positive-definite matrix (and thus also every real-valued symmetric positive-definite matrix) admits a unique Cholesky decomposition. 
By an explicit computation, it is then possible to show that the Cholesky decomposition of $\sigma_{\ell}$ takes the form $\sigma_{\ell}=\Lambda_{\ell} \Lambda_{\ell}^{t}$, where

$$
\begin{aligned}
\Lambda_{\ell} & =\left(\begin{array}{ccccc}
\frac{\sqrt{\lambda_{\ell}}}{\sqrt{2}} & 0 & 0 & 0 & 0 \\
0 & \frac{\sqrt{\lambda_{\ell}}}{\sqrt{2}} & 0 & 0 & 0 \\
0 & 0 & \frac{\sqrt{\lambda_{\ell}} \sqrt{3 \lambda_{\ell}-2}}{2 \sqrt{2}} & 0 & 0 \\
0 & 0 & 0 & \frac{\sqrt{\lambda_{\ell}} \sqrt{\lambda_{\ell}-2}}{2 \sqrt{2}} & 0 \\
0 & 0 & \frac{\sqrt{\lambda_{\ell}}\left(\lambda_{\ell}+2\right)}{2 \sqrt{2} \sqrt{3 \lambda_{\ell}-2}} & 0 & \frac{\lambda_{\ell} \sqrt{\lambda_{\ell}-2}}{\sqrt{3 \lambda_{\ell}-2}}
\end{array}\right) \\
& =:\left(\begin{array}{ccccc}
\lambda_{1} & 0 & 0 & 0 & 0 \\
0 & \lambda_{1} & 0 & 0 & 0 \\
0 & 0 & \lambda_{3} & 0 & 0 \\
0 & 0 & 0 & \lambda_{4} & 0 \\
0 & 0 & \lambda_{2} & 0 & \lambda_{5}
\end{array}\right) ;
\end{aligned}
$$

in the last expression, for notational simplicity we have omitted the dependence of the $\lambda_{i} \mathrm{~s}$ on $\ell$. The matrix is block diagonal, because under isotropy the gradient components are independent from the Hessian when evaluated at the same point (see, e.g., [1], Section 5.5). We can hence define a 5-dimensional standard Gaussian vector

$$
Y(x)=\left(Y_{1}(x), Y_{2}(x), Y_{3}(x), Y_{4}(x), Y_{5}(x)\right)
$$

with independent components such that

$$
\begin{aligned}
& \left(e_{1}^{x} f_{\ell}(x), e_{2}^{x} f_{\ell}(x), e_{1}^{x} e_{1}^{x} f_{\ell}(x), e_{1}^{x} e_{2}^{x} f_{\ell}(x), e_{2}^{x} e_{2}^{x} f_{\ell}(x)\right) \\
& \quad=\Lambda_{\ell} Y(x) \\
& \quad=\left(\lambda_{1} Y_{1}(x), \lambda_{1} Y_{2}(x), \lambda_{3} Y_{3}(x), \lambda_{4} Y_{4}(x), \lambda_{5} Y_{5}(x)+\lambda_{2} Y_{3}(x)\right) .
\end{aligned}
$$

The expression that we need to expand can then be written as

$$
\begin{aligned}
{\left[e_{1}^{x} e_{1}^{x} f_{\ell}(x) e_{2}^{x} e_{2}^{x} f_{\ell}(x)-\left(e_{1}^{x} e_{2}^{x} f_{\ell}(x)\right)^{2}\right] } & \times \mathbb{I}_{\left\{e_{1}^{x} e_{1}^{x} f_{\ell}(x)+e_{2}^{x} e_{2}^{x} f_{\ell}(x) \leq-\lambda_{\ell} u\right\}} \delta_{\varepsilon}\left(e_{1}^{x} f_{\ell}(x), e_{2}^{x} f_{\ell}(x)\right) \\
= & {\left[\lambda_{3} Y_{3}(x)\left\{\lambda_{5} Y_{5}(x)+\lambda_{2} Y_{3}(x)\right\}-\left\{\lambda_{4} Y_{4}(x)\right\}^{2}\right] } \\
& \times \mathbb{I}_{\left\{\frac{\lambda_{3}}{\lambda} Y_{3}(x)+\frac{\lambda_{5}}{\lambda} Y_{5}(x)+\frac{\lambda_{2}}{\lambda} Y_{3}(x) \leq-u\right\}} \delta_{\varepsilon}\left(\lambda_{1} Y_{1}(x), \lambda_{1} Y_{2}(x)\right) .
\end{aligned}
$$


3.2. Second-order chaotic component. We need now to start computing the projection coefficients on second-order Wiener chaoses. Our notation is as follows; we write $h_{i j}, i, j=1, \ldots, 5, i \neq j$, for the projections on terms of the form $H_{1}\left(Y_{i}\right) H_{1}\left(Y_{j}\right)=Y_{i} Y_{j}$, that is, we define

$$
\begin{aligned}
h_{i j}(u ; \ell)= & \lim _{\varepsilon \rightarrow 0} \mathbb{E}\left[\left[\lambda_{3} Y_{3}\left\{\lambda_{5} Y_{5}+\lambda_{2} Y_{3}\right\}-\left(\lambda_{4} Y_{4}\right)^{2}\right]\right. \\
& \left.\times \mathbb{1}_{\left\{\frac{\lambda_{2}+\lambda_{3}}{\lambda} Y_{3}+\frac{\lambda_{5}}{\lambda} Y_{5} \leq-u\right\}} \delta_{\varepsilon}\left(\lambda_{1} Y_{1}, \lambda_{1} Y_{2}\right) Y_{i} Y_{j}\right]
\end{aligned}
$$

on the other hand, we write $k_{i}, i=1, \ldots, 5$, for the projection on terms of the form $H_{2}\left(Y_{i}\right)$, that is, we define

$$
\begin{aligned}
k_{i}(u ; \ell)= & \lim _{\varepsilon \rightarrow 0} \mathbb{E}\left[\left[\lambda_{3} Y_{3}\left\{\lambda_{5} Y_{5}+\lambda_{2} Y_{3}\right\}-\left(\lambda_{4} Y_{4}\right)^{2}\right]\right. \\
& \left.\times \mathbb{1}_{\left\{\frac{\lambda_{2}+\lambda_{3}}{\lambda} Y_{3}+\frac{\lambda_{5}}{\lambda} Y_{5} \leq-u\right\}} \delta_{\varepsilon}\left(\lambda_{1} Y_{1}, \lambda_{1} Y_{2}\right) H_{2}\left(Y_{i}\right)\right] .
\end{aligned}
$$

The second-order chaotic component of the Euler-Poincaré characteristic is then given by

$$
\begin{aligned}
\operatorname{Proj} & {\left[\chi\left(A_{u}\left(f_{\ell} ; \mathbb{S}^{2}\right)\right) \mid 2\right] } \\
& =\sum_{i=1}^{5} \sum_{j=1}^{i} h_{i j}(u ; \ell) \int_{\mathbb{S}^{2}} Y_{i}(x) Y_{j}(x) d x+\frac{1}{2} \sum_{i=1}^{5} k_{i}(u ; \ell) \int_{\mathbb{S}^{2}} H_{2}\left(Y_{i}(x)\right) d x .
\end{aligned}
$$

The following proposition provides analytic expressions for the coefficients $h_{i j}$ and $k_{i}$.

PROpOsition 5. (a) All coefficients $h_{i j}(u ; \ell)$ are identically zero, unless $(i, j)=(3,5)$, that is,

$$
h_{i j}(u ; \ell)=\sqrt{\lambda_{\ell}} \sqrt{\lambda_{\ell}-2} \frac{\Phi(-u)\left(3 \lambda_{\ell}-2\right)+u \phi(u)\left[2+\lambda_{\ell}\left(u^{2}+1\right)\right]}{2 \sqrt{2} \pi\left(3 \lambda_{\ell}-2\right)} \delta_{i}^{3} \delta_{j}^{5} .
$$

(b) For the $k_{i}$ coefficients, we have

$$
\begin{aligned}
& k_{1}(u ; \ell)=k_{2}(u ; \ell)=-\frac{2 \Phi(-u)+\lambda_{\ell} u \phi(u)}{4 \pi}, \\
& k_{3}(u ; \ell)=\Phi(-u) \frac{\lambda_{\ell}+2}{4 \pi}+\lambda_{\ell} \frac{2+\lambda_{\ell}\left(u^{2}+1\right)}{2 \pi\left(3 \lambda_{\ell}-2\right)} u \phi(u), \\
& k_{4}(u ; \ell)=-\Phi(-u) \frac{\lambda_{\ell}-2}{4 \pi}, \quad k_{5}(u ; \ell)=\left(\lambda_{\ell}-2\right) \frac{\lambda_{\ell}\left(u^{2}+1\right)+2}{4 \pi\left(3 \lambda_{\ell}-2\right)} u \phi(u) .
\end{aligned}
$$

The proof of Proposition 5 is postponed to Section 5. From Proposition 5, it is then immediate to obtain the following expression:

$$
\operatorname{Proj}\left[\chi\left(A_{u}\left(f_{\ell} ; \mathbb{S}^{2}\right)\right) \mid 2\right]=h_{35}(u ; \ell) A_{35}(\ell)+\frac{1}{2} \sum_{i=1}^{5} k_{i}(u ; \ell) B_{i}(\ell),
$$


where

$$
A_{i j}(\ell)=\int_{\mathbb{S}^{2}} Y_{i}(x) Y_{j}(x) d x, \quad B_{i}(\ell)=\int_{\mathbb{S}^{2}} H_{2}\left(Y_{i}(x)\right) d x .
$$

Our next step is then to investigate the behaviour of these integrals of stochastic processes; this task is accomplished in the following lemma.

Proposition 6. We have that

$$
A_{35}(\ell)=4 \pi \frac{\sqrt{2}}{3} \sum_{m=-\ell}^{\ell}\left\{\left|a_{\ell m}\right|^{2}-1\right\}\left[-\frac{1}{\ell}+\frac{3 m}{\ell^{2}}-\frac{2 m^{3}}{\ell^{4}}\right]+r_{0}(\ell),
$$

and moreover,

$$
\begin{aligned}
& B_{1}(\ell)=4 \pi \sum_{m=-\ell}^{\ell}\left\{\left|a_{\ell m}\right|^{2}-1\right\}\left[\frac{1}{\ell}-\frac{m}{\ell^{2}}\right]+r_{1}(\ell), \\
& B_{2}(\ell)=4 \pi \sum_{m=-\ell}^{\ell}\left\{\left|a_{\ell m}\right|^{2}-1\right\} \frac{m}{\ell^{2}}+r_{2}(\ell), \\
& B_{3}(\ell)=4 \pi \sum_{m=-\ell}^{\ell}\left\{\left|a_{\ell m}\right|^{2}-1\right\}\left[\frac{4}{3 \ell}-\frac{2 m}{\ell^{2}}+\frac{2 m^{3}}{3 \ell^{4}}\right]+r_{3}(\ell), \\
& B_{4}(\ell)=4 \pi \times 2 \sum_{m=-\ell}^{\ell}\left\{\left|a_{\ell m}\right|^{2}-1\right\}\left[\frac{m}{\ell^{2}}-\frac{m^{3}}{\ell^{4}}\right]+r_{4}(\ell), \\
& B_{5}(\ell)=4 \pi \times \frac{1}{6} \sum_{m=-\ell}^{\ell}\left\{\left|a_{\ell m}\right|^{2}-1\right\}\left[\frac{1}{\ell}+\frac{8 m^{3}}{\ell^{4}}\right]+r_{5}(\ell),
\end{aligned}
$$

where $\sqrt{\mathbb{E}\left[r_{i}(\ell)\right]^{2}}=O\left(\ell^{-1}\right)$, for all $i=0, \ldots, 5$.

The proof of Proposition 6 is also postponed to Section 5. We are now in the position to conclude the main proof of this section.

Proof of TheOREM 1. A simple rewriting of the results from Proposition 5 yields

$$
h_{35}(u ; \ell)=\ell^{2}\left\{\frac{\Phi(-u)}{2 \sqrt{2} \pi}+u \phi(u) \frac{u^{2}+1}{6 \sqrt{2} \pi}\right\}+O(\ell)
$$

and also

$$
k_{1}(u ; \ell)=k_{2}(u ; \ell)=-\ell^{2} \frac{u \phi(u)}{4 \pi}+O(\ell),
$$




$$
\begin{aligned}
& k_{3}(u ; \ell)=\ell^{2}\left\{\frac{\Phi(-u)}{4 \pi}+u \phi(u) \frac{u^{2}+1}{6 \pi}\right\}+O(\ell), \\
& k_{4}(u ; \ell)=-\ell^{2} \frac{\Phi(-u)}{4 \pi}+O(\ell), \quad k_{5}(u ; \ell)=\ell^{2} u \phi(u) \frac{u^{2}+1}{12 \pi}+O(\ell),
\end{aligned}
$$

where the terms $O(\ell)$ are all uniform over $u$. Now replacing the expressions which were derived in Proposition 6, we can hence write down the projection on the second-order Wiener chaos as follows:

$$
\begin{aligned}
\operatorname{Proj}[ & \left.\chi\left(A_{u}\left(f_{\ell} ; \mathbb{S}^{2}\right)\right) \mid 2\right] \\
= & \ell^{2}\left\{\frac{\Phi(-u)}{2 \sqrt{2} \pi}+u \phi(u) \frac{u^{2}+1}{6 \sqrt{2} \pi}\right\} \\
& \times\left\{4 \pi \frac{\sqrt{2}}{3} \sum_{m=-\ell}^{\ell}\left\{\left|a_{\ell m}\right|^{2}-1\right\}\left[-\frac{1}{\ell}+\frac{3 m}{\ell^{2}}-\frac{2 m^{3}}{\ell^{4}}\right]\right\} \\
& -\frac{1}{2} \ell^{2} \frac{u \phi(u)}{4 \pi}\left\{4 \pi \sum_{m=-\ell}^{\ell}\left\{\left|a_{\ell m}\right|^{2}-1\right\}\left[\frac{1}{\ell}-\frac{m}{\ell^{2}}\right]\right\} \\
& -\frac{1}{2} \ell^{2} \frac{u \phi(u)}{4 \pi}\left\{4 \pi \sum_{m=-\ell}^{\ell}\left\{\left|a_{\ell m}\right|^{2}-1\right\} \frac{m}{\ell^{2}}\right\} \\
& +\frac{1}{2} \ell^{2}\left\{\frac{\Phi(-u)}{4 \pi}+u \phi(u) \frac{u^{2}+1}{6 \pi}\right\} \\
& \times\left\{4 \pi \sum_{m=-\ell}^{\ell}\left\{\left|a_{\ell m}\right|^{2}-1\right\}\left[\frac{4}{3 \ell}-\frac{2 m}{\ell^{2}}+\frac{2 m^{3}}{3 \ell^{4}}\right]\right\} \\
& -\frac{1}{2} \ell^{2} \frac{\Phi(-u)}{4 \pi}\left\{4 \pi \times 2 \sum_{m=-\ell}^{\ell}\left\{\left|a_{\ell m}\right|^{2}-1\right\}\left[\frac{m}{\ell^{2}}-\frac{m^{3}}{\ell^{4}}\right]\right\} \\
& +\frac{1}{2} \ell^{2} u \phi(u) \frac{u^{2}+1}{12 \pi}\left\{4 \pi \frac{1}{6} \sum_{m=-\ell}^{\ell}\left\{\left|a_{\ell m}\right|^{2}-1\right\}\left[\frac{1}{\ell}+\frac{8 m^{3}}{\ell^{4}}\right]\right\}+R_{1}(\ell),
\end{aligned}
$$

where the remainder term $R_{1}(\ell)$ is such that $\sqrt{\mathbb{E}\left[R_{1}(\ell)\right]^{2}}=O(\ell)$, again uniformly over $u$. We now show that all terms which include the Gaussian cumulative distribution function cancel; more precisely, performing some simple manipulations it is immediate to note that

$$
\begin{gathered}
\ell^{2} \frac{\Phi(-u)}{2 \sqrt{2} \pi} A_{35}(\ell)+\frac{1}{2} \ell^{2} \frac{\Phi(-u)}{4 \pi} B_{3}(\ell)-\frac{1}{2} \ell^{2} \frac{\Phi(-u)}{4 \pi} B_{4}(\ell) \\
=2 \ell^{2} \frac{\Phi(-u)}{3}\left\{\sum_{m=-\ell}^{\ell}\left\{\left|a_{\ell m}\right|^{2}-1\right\}\left[-\frac{1}{\ell}+\frac{3 m}{\ell^{2}}-\frac{2 m^{3}}{\ell^{4}}\right]\right\}
\end{gathered}
$$




$$
\begin{aligned}
& +\frac{1}{2} \ell^{2} \Phi(-u)\left\{\sum_{m=-\ell}^{\ell}\left\{\left|a_{\ell m}\right|^{2}-1\right\}\left[\frac{4}{3 \ell}-\frac{2 m}{\ell^{2}}+\frac{2 m^{3}}{3 \ell^{4}}\right]\right\} \\
& -\ell^{2} \Phi(-u)\left\{\sum_{m=-\ell}^{\ell}\left\{\left|a_{\ell m}\right|^{2}-1\right\}\left[\frac{m}{\ell^{2}}-\frac{m^{3}}{\ell^{4}}\right]\right\}+R_{2}(\ell)=R_{2}(\ell),
\end{aligned}
$$

where again the remainder term is uniformly bounded by $O(\ell)$ in the mean-square norm. Rearranging the remaining terms, we thus obtain

$$
\begin{aligned}
\operatorname{Proj}[ & \left.\chi\left(A_{u}\left(f_{\ell} ; \mathbb{S}^{2}\right)\right) \mid 2\right] \\
= & \ell^{2} u \phi(u) \frac{u^{2}+1}{6 \sqrt{2} \pi} A_{35}(\ell)-\frac{1}{2} \ell^{2} u \phi(u) \frac{1}{4 \pi}\left\{B_{1}(\ell)+B_{2}(\ell)\right\}+R(\ell) \\
= & \ell^{2} u \phi(u) \frac{u^{2}+1}{6 \sqrt{2} \pi}\left\{4 \pi \frac{\sqrt{2}}{3} \sum_{m=-\ell}^{\ell}\left\{\left|a_{\ell m}\right|^{2}-1\right\}\left[-\frac{1}{\ell}+\frac{3 m}{\ell^{2}}-\frac{2 m^{3}}{\ell^{4}}\right]\right\} \\
& -\frac{1}{2} \ell^{2} u \phi(u) \frac{1}{4 \pi}\left\{4 \pi \sum_{m=-\ell}^{\ell}\left\{\left|a_{\ell m}\right|^{2}-1\right\}\left[\frac{1}{\ell}-\frac{m}{\ell^{2}}\right]\right\} \\
& -\frac{1}{2} \ell^{2} u \phi(u) \frac{1}{4 \pi}\left\{4 \pi \sum_{m=-\ell}^{\ell}\left\{\left|a_{\ell m}\right|^{2}-1\right\} \frac{m}{\ell^{2}}\right\} \\
& +\frac{1}{2} \ell^{2}\left\{u \phi(u) \frac{u^{2}+1}{6 \pi}\right\}\left\{\frac{1}{4 \pi} \sum_{m=-\ell}^{\ell}\left\{\left|a_{\ell m}\right|^{2}-1\right\}\left[\frac{4}{3 \ell}-\frac{2 m}{\ell^{2}}+\frac{2 m^{3}}{3 \ell^{4}}\right]\right\} \\
& +\frac{1}{2} \ell^{2} u \phi(u) \frac{u^{2}+1}{12 \pi}\left\{4 \pi \frac{1}{6} \sum_{m=-\ell}^{\ell}\left\{\left|a_{\ell m}\right|^{2}-1\right\}\left[\frac{1}{\ell}+\frac{8 m^{3}}{\ell^{4}}\right]\right\}+R(\ell) \\
& +\frac{1}{2} \ell^{2} u \phi(u) \frac{u^{2}+1}{18}\left\{\frac{1}{\ell} \sum_{m=-\ell}^{\ell}\left\{\left|a_{\ell m}\right|^{2}-1\right\}\right\}+R(\ell) \\
= & \ell^{2} u \phi(u)\left(u^{2}+1\right) \frac{2}{9}\left\{-\frac{1}{\ell} \sum_{m=-\ell}^{\ell}\left\{\left|a_{\ell m}\right|^{2}-1\right\}\right\} \\
& \left.-\frac{\ell}{m=-\ell}\left\{\left|a_{\ell m}\right|^{2}-1\right\} \frac{1}{\ell}\right\} \\
& \\
&
\end{aligned}
$$




$$
=\ell u \phi(u) \frac{u^{2}-1}{4} \sum_{m=-\ell}^{\ell}\left\{\left|a_{\ell m}\right|^{2}-1\right\}+R(\ell),
$$

where $\sqrt{\mathbb{E} R^{2}(\ell)}=O(\ell)$, as claimed.

4. Variance and quantitative central limit theorem. In this section, we prove Theorem 2. Our first result is the following.

LEMMA 7. As $\ell \rightarrow \infty$, for all $u \neq 0$ we have that

$$
\frac{\operatorname{Var}\left[\operatorname{Proj}\left[\chi\left(A_{u}\left(f_{\ell} ; \mathbb{S}^{2}\right)\right) \mid 2\right]\right]}{\operatorname{Var}\left[\chi\left(A_{u}\left(f_{\ell} ; \mathbb{S}^{2}\right)\right)\right]}=1+O\left(\frac{\log \ell}{\ell}\right) .
$$

PROOF. In [5, 7], it is shown that, for all $u \neq 0$

$$
\operatorname{Var}\left[\chi\left(A_{u}\left(f_{\ell} ; \mathbb{S}^{2}\right)\right)\right]=\frac{1}{4} \ell^{3}\left\{u \phi(u)\left(u^{2}-1\right)\right\}^{2}+O\left(\ell^{2} \log \ell\right),
$$

the error term being uniform over $u$. In view of the form of $\operatorname{Proj}\left[\chi\left(A_{u}\left(f_{\ell}\right.\right.\right.$; $\left.\left.\left.\mathbb{S}^{2}\right)\right) \mid 2\right]$, we need only consider the asymptotic variance of $\sum_{m=-\ell}^{\ell}\left\{\left|a_{\ell m}\right|^{2}-1\right\}$; the details are trivial, but we report them for completeness. Recall first that

$$
\left|a_{\ell m}\right|^{2}=\left\{\operatorname{Re}\left(a_{\ell m}\right)\right\}^{2}+\left\{\operatorname{Im}\left(a_{\ell m}\right)\right\}^{2}=\left|a_{\ell,-m}\right|^{2},
$$

where $\operatorname{Re}\left(a_{\ell m}\right), \operatorname{Im}\left(a_{\ell m}\right)$ are zero-mean, independent Gaussian variables with variance $\frac{1}{2}$; on the other hand, $a_{\ell 0}$ follows a standard $N(0,1)$ Gaussian distribution. We can thus write

$$
\begin{aligned}
\sum_{m=-\ell}^{\ell}\left\{\left|a_{\ell m}\right|^{2}-1\right\}= & \left\{\left|a_{\ell 0}\right|^{2}-1\right\}+2 \sum_{m=1}^{\ell}\left\{\left|a_{\ell m}\right|^{2}-1\right\} \\
= & \left\{\left|a_{\ell 0}\right|^{2}-1\right\}+\sum_{m=1}^{\ell}\left\{\operatorname{Re}\left|\sqrt{2} a_{\ell m}\right|^{2}-1\right\} \\
& +\sum_{m=1}^{\ell}\left\{\operatorname{Im}\left|\sqrt{2} a_{\ell m}\right|^{2}-1\right\} .
\end{aligned}
$$

Now note that $\left|a_{\ell 0}\right|^{2}, \operatorname{Re}\left|\sqrt{2} a_{\ell m}\right|^{2}, \operatorname{Im}\left|\sqrt{2} a_{\ell m}\right|^{2}, m=1, \ldots, \ell$ are a set of $2 \ell+1$ independent variables distributed according to a $\chi_{1}^{2}$ with one degree of freedom; it follows immediately that

$$
\operatorname{Var}\left[\sum_{m=-\ell}^{\ell}\left\{\left|a_{\ell m}\right|^{2}-1\right\}\right]=2(2 \ell+1) .
$$


Thus

$$
\begin{aligned}
& \lim _{\ell \rightarrow \infty} \frac{\operatorname{Var}\left[\operatorname{Proj}\left[\chi\left(A_{u}\left(f_{\ell} ; \mathbb{S}^{2}\right)\right) \mid 2\right]\right]}{\frac{1}{4} \ell^{3}\left\{u \phi(u)\left(u^{2}-1\right)\right\}^{2}} \\
& \quad=\lim _{\ell \rightarrow \infty} \frac{\operatorname{Var}\left[\frac{1}{4} \ell u \phi(u)\left(u^{2}-1\right) \sum_{m=-\ell}^{\ell}\left\{\left|a_{\ell m}\right|^{2}-1\right\}\right]}{\frac{1}{4} \ell^{3}\left\{u \phi(u)\left(u^{2}-1\right)\right\}^{2}} \\
& \quad=\frac{1}{4} \lim _{\ell \rightarrow \infty} \frac{\operatorname{Var}\left[\sum_{m=-\ell}^{\ell}\left\{\left|a_{\ell m}\right|^{2}-1\right\}\right]}{\ell}=1,
\end{aligned}
$$

and the result we claimed follows immediately.

Proof of THEOREM 2. We recall that the Wasserstein distance between random variables $X, Y$ is defined by

$$
d_{W}(X, Y):=\sup _{h \in L i p(1)}|\mathbb{E} h(X)-\mathbb{E} h(Y)| ;
$$

also, $d_{W}(X, Y) \leq \sqrt{\mathbb{E}|X-Y|^{2}}$, that is, Wasserstein distance is always bounded by the $L^{2}$-metric; see [25] for further characterizations and details. By the triangle inequality, we have

$$
\begin{aligned}
& d_{W}\left(\frac{\chi\left(A_{u}\left(f_{\ell} ; \mathbb{S}^{2}\right)\right)-\mathbb{E}\left[\chi\left(A_{u}\left(f_{\ell} ; \mathbb{S}^{2}\right)\right)\right]}{\sqrt{\operatorname{Var}\left[\chi\left(A_{u}\left(f_{\ell} ; \mathbb{S}^{2}\right)\right)\right]}}, Z\right) \\
& \leq d_{W}\left(\frac{\chi\left(A_{u}\left(f_{\ell} ; \mathbb{S}^{2}\right)\right)-\mathbb{E}\left[\chi\left(A_{u}\left(f_{\ell} ; \mathbb{S}^{2}\right)\right)\right]}{\sqrt{\operatorname{Var}\left[\chi\left(A_{u}\left(f_{\ell} ; \mathbb{S}^{2}\right)\right)\right]}}, \frac{\operatorname{Proj}\left[\chi\left(A_{u}\left(f_{\ell} ; \mathbb{S}^{2}\right)\right) \mid 2\right]}{\sqrt{\operatorname{Var}\left[\chi\left(A_{u}\left(f_{\ell} ; \mathbb{S}^{2}\right)\right)\right]}}\right) \\
& +d_{W}\left(\frac{\operatorname{Proj}\left[\chi\left(A_{u}\left(f_{\ell} ; \mathbb{S}^{2}\right)\right) \mid 2\right]}{\sqrt{\operatorname{Var}\left[\chi\left(A_{u}\left(f_{\ell} ; \mathbb{S}^{2}\right)\right)\right]}}, Z\right) \\
& =d_{W}\left(\frac{\operatorname{Proj}\left[\chi\left(A_{u}\left(f_{\ell} ; \mathbb{S}^{2}\right)\right) \mid 2\right]}{\sqrt{\operatorname{Var}\left[\chi\left(A_{u}\left(f_{\ell} ; \mathbb{S}^{2}\right)\right)\right]}}, Z\right)+O\left(\sqrt{\frac{\log \ell}{\ell}}\right),
\end{aligned}
$$

because

$$
\begin{aligned}
& \mathbb{E}\left\{\frac{\chi\left(A_{u}\left(f_{\ell} ; \mathbb{S}^{2}\right)\right)-\mathbb{E}\left[\chi\left(A_{u}\left(f_{\ell} ; \mathbb{S}^{2}\right)\right)\right]-\operatorname{Proj}\left[\chi\left(A_{u}\left(f_{\ell} ; \mathbb{S}^{2}\right)\right) \mid 2\right]}{\sqrt{\operatorname{Var}\left[\chi\left(A_{u}\left(f_{\ell} ; \mathbb{S}^{2}\right)\right)\right]}}\right\}^{2} \\
& =O\left(\frac{\log \ell}{\ell}\right),
\end{aligned}
$$

uniformly over $u$. By a similar argument,

$$
d_{W}\left(\frac{\operatorname{Proj}\left[\chi\left(A_{u}\left(f_{\ell} ; \mathbb{S}^{2}\right)\right) \mid 2\right]}{\sqrt{\operatorname{Var}\left[\chi\left(A_{u}\left(f_{\ell} ; \mathbb{S}^{2}\right)\right)\right]}}, Z\right)=d_{W}\left(F_{\ell}(u) ; Z\right)+O\left(\sqrt{\frac{1}{\ell}}\right)
$$


where we wrote for notational simplicity

$$
F_{\ell}(u):=\frac{\frac{\lambda_{\ell}}{2}\left\{H_{1}(u) H_{2}(u) \phi(u)\right\} \frac{1}{2 \ell+1} \sum_{m=-\ell}^{\ell}\left\{\left|a_{\ell m}\right|^{2}-1\right\}}{\sqrt{\operatorname{Var}\left[\chi\left(A_{u}\left(f_{\ell} ; \mathbb{S}^{2}\right)\right)\right]}} ;
$$

from Corollary 5.2.10 in [25] we have

$$
\begin{aligned}
d_{W}\left(F_{\ell}(u), Z\right) & \leq \sqrt{\frac{2\left(\mathbb{E} F_{\ell}^{4}(u)-3\left[\mathbb{E} F_{\ell}^{2}(u)\right]^{2}\right)}{3 \pi\left[\mathbb{E} F_{\ell}^{2}(u)\right]^{2}}}+\sqrt{\frac{\sqrt{\frac{2}{\pi}}\left(\mathbb{E} F_{\ell}^{2}(u)-1\right)}{\mathbb{E} F_{\ell}^{2}(u) \vee 1}} \\
& =\sqrt{\frac{2\left(\mathbb{E} F_{\ell}^{4}(u)-3\left[\mathbb{E} F_{\ell}^{2}(u)\right]^{2}\right)}{3 \pi\left[\mathbb{E} F_{\ell}^{2}(u)\right]^{2}}}+O\left(\sqrt{\frac{\log \ell}{\ell}}\right),
\end{aligned}
$$

in view of Lemma 7. To complete the proof, it suffices to notice that for every fixed $u, \mathbb{E} F_{\ell}^{4}(u)-3\left[\mathbb{E} F_{\ell}^{2}(u)\right]^{2}$ is the fourth-order cumulant of the sample average of $2 \ell+1$ independent random variables with finite moments of all order; it is then a standard exercise to show that this quantity is $O\left(\ell^{-1}\right)$, which completes the proof.

REMARK 8. The theorem can be generalized to joint convergence for every fixed set of threshold levels $\left(u_{1}, \ldots, u_{p}\right), p \in \mathbb{N}$; details are trivial, and hence omitted. A more interesting possibility would be to investigate a functional central limit theorem over $u$; this extension seems possible, but we do not consider it here for brevity's sake.

\section{Proofs of technical results.}

5.1. Proof of Proposition 5. Let $Y$ be a standard random variable; for the projection coefficients of the Dirac's delta function (which are given for instance in [25], Chapter 1; see also [18]), we introduce the following notation:

$$
\varphi_{a}(\ell)=\lim _{\varepsilon \rightarrow 0} \mathbb{E}\left[H_{a}(Y) \delta_{\varepsilon}\left(\lambda_{1} Y\right)\right], \quad a=0,1,2 .
$$

We also use $\theta_{a b}$ to denote projection coefficients involving two random variables $Y_{a}, Y_{b}$ and $\psi_{a b c d}(u)$ to denote those coefficients that involve four, that is, we set

$$
\theta_{a b}(u)=\mathbb{E}\left[Y_{a} Y_{b} \mathbb{1}_{\left\{\frac{\lambda_{2}+\lambda_{3}}{\lambda} Y_{3}+\frac{\lambda_{5}}{\lambda} Y_{5} \leq-u\right\}}\right], \quad a, b=3,4,5,
$$

and

$$
\psi_{a b c d}(u)=\mathbb{E}\left[Y_{a} Y_{b} Y_{c} Y_{d} \mathbb{1}_{\left\{\frac{\lambda_{2}+\lambda_{3}}{\lambda} Y_{3}+\frac{\lambda_{5}}{\lambda} Y_{5} \leq-u\right\}}\right], \quad a, b, c, d=3,4,5 .
$$

The exact behaviour of these coefficients as a function of the level $u$ is given in the three lemmas to follow; the proofs are elementary, albeit long and tedious, 
exercises in the computation of Gaussian moments and convolutions, and hence they are omitted (more details can be found in the arXiv preprint version of this paper). We recall once again that we use $\phi(\cdot)$ and $\Phi(\cdot)$ to denote as usual the density and distribution function of a standard Gaussian random variable.

LEMMA 9. We have

$$
\varphi_{a}(\ell)= \begin{cases}\frac{1}{\sqrt{2 \pi} \lambda_{1}}, & a=0 \\ 0, & a=1, \\ -\frac{1}{\sqrt{2 \pi} \lambda_{1}}, & a=2 .\end{cases}
$$

LEMMA 10. We have that

$$
\begin{aligned}
& \theta_{33}(u)=\Phi(-u)+\frac{2 \lambda_{\ell}}{3 \lambda_{\ell}-2} u \phi(u), \quad \theta_{35}(u)=\sqrt{2} \frac{\sqrt{\lambda_{\ell}} \sqrt{\lambda_{\ell}-2}}{3 \lambda_{\ell}-2} u \phi(u), \\
& \theta_{44}(u)=\Phi(-u) .
\end{aligned}
$$

The computation of expected values involving four moments is clearly more challenging and is detailed in the lemma below.

LEMMA 11. (a) The expression for the coefficients involving only $Y_{3}$ or $Y_{4}$ is equal to

$$
\psi_{3333}(u)=3 \Phi(-u)+4 \lambda_{\ell} \frac{\lambda_{\ell}\left(u^{2}+6\right)-6}{\left(3 \lambda_{\ell}-2\right)^{2}} u \phi(u), \quad \psi_{4444}(u)=3 \Phi(-u) .
$$

(b) The expression for coefficients involving cross products of $Y_{3}$ and $Y_{5}$ are equal to

$$
\begin{aligned}
& \psi_{3355}(u)=\Phi(-u)+\frac{4+2 u^{2} \lambda_{\ell}\left(\lambda_{\ell}-2\right)+3 \lambda_{\ell}^{2}}{\left(3 \lambda_{\ell}-2\right)^{2}} u \phi(u), \\
& \psi_{3555}(u)=\sqrt{2}\left(\lambda_{\ell} u^{2}-2 u^{2}+6 \lambda_{\ell}\right) \frac{\sqrt{\lambda_{\ell}} \sqrt{\lambda_{\ell}-2}}{\left(3 \lambda_{\ell}-2\right)^{2}} u \phi(u), \\
& \psi_{3335}(u)=\sqrt{2}\left(2 \lambda_{\ell} u^{2}+3 \lambda_{\ell}-6\right) \frac{\sqrt{\lambda_{\ell}} \sqrt{\lambda_{\ell}-2}}{\left(3 \lambda_{\ell}-2\right)^{2}} u \phi(u) .
\end{aligned}
$$

(c) The expression for coefficients involving cross-products with $Y_{4}$ are as follows:

$$
\begin{aligned}
& \psi_{3344}(u)=\Phi(-u)+\frac{2 \lambda_{\ell}}{3 \lambda_{\ell}-2} u \phi(u), \quad \psi_{4455}(u)=\Phi(-u)+\frac{\lambda_{\ell}-2}{3 \lambda_{\ell}-2} u \phi(u), \\
& \psi_{3445}(u)=\sqrt{2} \frac{\sqrt{\lambda_{\ell}} \sqrt{\lambda_{\ell}-2}}{3 \lambda_{\ell}-2} u \phi(u) .
\end{aligned}
$$


(d) The following remaining terms are identically zero:

$$
\psi_{3334}(u)=\psi_{3345}(u)=\psi_{3444}(u)=\psi_{3455}(u)=\psi_{4445}(u)=0 .
$$

End of the proof of Proposition 5. We are now in the position to complete the proof of the proposition. First note that, in view of Lemma 9, we immediately have $h_{1 j}(u ; \ell)=0$ for all $j>1$ and $h_{2 j}(u ; \ell)=0$ for all $j>2$ since $\varphi_{1}(\ell)=0$. Moreover, some standard algebraic computations yield

$$
h_{34}(u ; \ell)=\left[\lambda_{3} \lambda_{5} \psi_{3345}(u)+\lambda_{2} \lambda_{3} \psi_{3334}(u)-\lambda_{4}^{2} \psi_{3444}(u)\right] \varphi_{0}^{2}(\ell)=0
$$

PROOF.

$$
\begin{aligned}
h_{35}(u ; \ell) & =\left[\lambda_{3} \lambda_{5} \psi_{3355}(u)+\lambda_{2} \lambda_{3} \psi_{3335}(u)-\lambda_{4}^{2} \psi_{3445}(u)\right] \varphi_{0}^{2}(\ell) \\
& =\sqrt{\lambda_{\ell}} \sqrt{\lambda_{\ell}-2} \frac{\Phi(-u)\left(3 \lambda_{\ell}-2\right)+u \phi(u)\left[2+\lambda_{\ell}\left(u^{2}+1\right)\right]}{2 \sqrt{2} \pi\left(3 \lambda_{\ell}-2\right)}, \\
h_{45}(u ; \ell) & =\left[\lambda_{3} \lambda_{5} \psi_{3455}(u)+\lambda_{2} \lambda_{3} \psi_{3345}(u)-\lambda_{4}^{2} \psi_{4445}(u)\right] \varphi_{0}^{2}(\ell)=0 .
\end{aligned}
$$

The first part of the proposition is hence proved. For the second part, we can argue similarly and obtain

$$
\begin{aligned}
k_{1}(u ; \ell)= & k_{2}(u ; \ell)=\left[\lambda_{3} \lambda_{5} \theta_{35}(u)+\lambda_{2} \lambda_{3} \theta_{33}(u)-\lambda_{4}^{2} \theta_{44}(u)\right] \varphi(0) \varphi(2) \\
= & -\frac{2 \Phi(-u)+\lambda_{\ell} u \phi(u)}{4 \pi}, \\
k_{3}(u ; \ell)= & \left\{\left[\lambda_{3} \lambda_{5} \psi_{3335}(u)+\lambda_{2} \lambda_{3} \psi_{3333}(u)-\lambda_{4}^{2} \psi_{3344}(u)\right]\right. \\
& \left.-\left[\lambda_{3} \lambda_{5} \theta_{35}(u)+\lambda_{2} \lambda_{3} \theta_{33}(u)-\lambda_{4}^{2} \theta_{44}(u)\right]\right\} \varphi^{2}(0) \\
= & \frac{\Phi(-u)\left(\lambda_{\ell}+4\right)\left(3 \lambda_{\ell}-2\right)+8 \lambda_{\ell}\left(\lambda_{\ell}\left(u^{2}+1\right)+2\right) u \phi(u)}{4 \pi\left(3 \lambda_{\ell}-2\right)} \\
= & \Phi(-u) \frac{\lambda_{\ell}+4}{4 \pi}+\lambda_{\ell} \frac{\lambda_{\ell}\left(2 u^{2}+5\right)+2}{4 \pi\left(3 \lambda_{\ell}-2\right)} u \phi(u), \\
k_{4}(u ; \ell)= & \left\{\left[\lambda_{3} \lambda_{5} \psi_{3445}(u)+\lambda_{2} \lambda_{3} \psi_{3344}(u)-\lambda_{4}^{2} \psi_{4444}(u)\right]\right. \\
& \left.-\left[\lambda_{3} \lambda_{5} \theta_{35}(u)+\lambda_{2} \lambda_{3} \theta_{33}(u)-\lambda_{4}^{2} \theta_{44}(u)\right]\right\} \varphi^{2}(0) \\
= & -\Phi(-u) \frac{\lambda_{\ell}-2}{4 \pi},
\end{aligned}
$$

and finally

$$
\begin{aligned}
k_{5}(u ; \ell)= & \left\{\left[\lambda_{3} \lambda_{5} \psi_{3555}(u)+\lambda_{2} \lambda_{3} \psi_{3355}(u)-\lambda_{4}^{2} \psi_{4455}(u)\right]\right. \\
& \left.-\left[\lambda_{3} \lambda_{5} \theta_{35}(u)+\lambda_{2} \lambda_{3} \theta_{33}(u)-\lambda_{4}^{2} \theta_{44}(u)\right]\right\} \varphi^{2}(0) \\
= & \left(\lambda_{\ell}-2\right) \frac{\lambda_{\ell}\left(u^{2}+1\right)+2}{4 \pi\left(3 \lambda_{\ell}-2\right)} u \phi(u) .
\end{aligned}
$$


5.2. Proof of Proposition 6. We need first to introduce some more notation concerning the integrals of products of random eigenfunction and/or their derivatives. As before, we denote by $e_{a}^{x}, a=1,2$, the covariant derivative at $x \in \mathbb{S}^{2}$ with respect to the first or second variable $\theta, \varphi$. We have to deal with the following integrals of squares:

$$
\begin{aligned}
& I_{00}(\ell)=\int_{\mathbb{S}^{2}} f_{\ell}^{2}(x) d x, \quad I_{11}(\ell)=\int_{\mathbb{S}^{2}}\left\{e_{1}^{x} f_{\ell}(x)\right\}^{2} d x, \\
& I_{22}(\ell)=\int_{\mathbb{S}^{2}}\left\{e_{2}^{x} f_{\ell}(x)\right\}^{2} d x ;
\end{aligned}
$$

we shall also study the cross-product integral

$$
I_{0,22}(\ell)=\int_{\mathbb{S}^{2}} f_{\ell}(x) e_{2}^{x} e_{2}^{x} f_{\ell}(x) d x
$$

and finally we shall consider

$$
I_{12,12}(\ell)=\int_{\mathbb{S}^{2}}\left\{e_{1}^{x} e_{2}^{x} f_{\ell}(x)\right\}^{2} d x, \quad I_{22,22}(\ell)=\int_{\mathbb{S}^{2}}\left\{e_{2}^{x} e_{2}^{x} f_{\ell}(x)\right\}^{2} d x .
$$

Let us now show how the analysis of these 6 integrals will suffice for our needs. First note that, since

$$
Y_{5}(x)=\frac{1}{\lambda_{5}}\left\{e_{2}^{x} e_{2}^{x} f_{\ell}(x)-\frac{\lambda_{2}}{\lambda_{3}} e_{1}^{x} e_{1}^{x} f_{\ell}(x)\right\}
$$

and

$$
e_{1}^{x} e_{1}^{x} f_{\ell}(x)=-\lambda_{\ell} f_{\ell}(x)-e_{2}^{x} e_{2}^{x} f_{\ell}(x)
$$

we have

$$
\begin{aligned}
A_{35} & =\frac{1}{\lambda_{3} \lambda_{5}} \int_{\mathbb{S}^{2}} e_{1}^{x} e_{1}^{x} f_{\ell}(x)\left\{e_{2}^{x} e_{2}^{x} f_{\ell}(x)-\frac{\lambda_{2}}{\lambda_{3}} e_{1}^{x} e_{1}^{x} f_{\ell}(x)\right\} d x \\
& =-\frac{\lambda_{\ell}}{\lambda_{3} \lambda_{5}}\left\{1+2 \frac{\lambda_{2}}{\lambda_{3}}\right\} I_{0,22}(\ell)-\frac{\lambda_{\ell}^{2} \lambda_{2}}{\lambda_{3}^{2} \lambda_{5}} I_{00}(\ell)-\frac{1}{\lambda_{3} \lambda_{5}}\left\{1+\frac{\lambda_{2}}{\lambda_{3}}\right\} I_{22,22}(\ell) .
\end{aligned}
$$

Likewise

$$
\begin{aligned}
& B_{1}=\int_{\mathbb{S}^{2}} H_{2}\left(\frac{e_{1}^{x} f_{\ell}(x)}{\lambda_{1}}\right) d x=\frac{1}{\lambda_{1}^{2}} I_{11}(\ell)-4 \pi, \\
& B_{2}=\int_{\mathbb{S}^{2}} H_{2}\left(\frac{e_{2}^{x} f_{\ell}(x)}{\lambda_{1}}\right) d x=\frac{1}{\lambda_{1}^{2}} I_{22}(\ell)-4 \pi,
\end{aligned}
$$

so that these terms only require the investigation of integrals in (25). Finally, for the remaining terms it suffices to note that

$$
\begin{aligned}
& B_{3}=\int_{\mathbb{S}^{2}} H_{2}\left(\frac{e_{1}^{x} e_{1}^{x} f_{\ell}(x)}{\lambda_{3}}\right) d x=\frac{\lambda_{\ell}^{2}}{\lambda_{3}^{2}} I_{00}(\ell)+\frac{1}{\lambda_{3}^{2}} I_{22,22}(\ell)+\frac{2 \lambda_{\ell}}{\lambda_{3}^{2}} I_{0,22}(\ell)-4 \pi, \\
& B_{4}=\int_{\mathbb{S}^{2}} H_{2}\left(\frac{e_{1}^{x} e_{2}^{x} f_{\ell}(x)}{\lambda_{4}}\right) d x=\frac{1}{\lambda_{4}^{2}} I_{12,12}(\ell)-4 \pi
\end{aligned}
$$


and

$$
\begin{aligned}
B_{5} & =\int_{\mathbb{S}^{2}} H_{2}\left(\frac{1}{\lambda_{5}} e_{2}^{x} e_{2}^{x} f_{\ell}(x)-\frac{\lambda_{2}}{\lambda_{3} \lambda_{5}} e_{1}^{x} e_{1}^{x} f_{\ell}(x)\right) d x \\
& =\frac{1}{\lambda_{5}^{2}}\left(1+\frac{\lambda_{2}}{\lambda_{3}}\right)^{2} I_{22,22}(\ell)+\frac{\lambda_{\ell}^{2} \lambda_{2}^{2}}{\lambda_{3}^{2} \lambda_{5}^{2}} I_{00}(\ell)+2 \frac{\lambda_{\ell} \lambda_{2}}{\lambda_{3} \lambda_{5}^{2}}\left(1+\frac{\lambda_{2}}{\lambda_{3}}\right) I_{0,22}(\ell)-4 \pi .
\end{aligned}
$$

To conclude the proof of the proposition, it is enough to write these integrals explicitly in terms of the spherical harmonic coefficients $\left\{a_{\ell m}\right\}$. This task is accomplished in the following lemma.

LEMMA 12. (a) For the integrals of square terms, we have that

$$
\begin{aligned}
I_{00}(\ell) & =\frac{4 \pi}{2 \ell+1} a_{\ell 0}^{2}+\frac{2 \cdot 4 \pi}{2 \ell+1} \sum_{m>0}\left|a_{\ell m}\right|^{2}=\frac{4 \pi}{2 \ell+1} \sum_{m=-\ell}^{\ell}\left|a_{\ell m}\right|^{2} \\
I_{11}(\ell) & =4 \pi a_{\ell 0}^{2} \frac{\lambda_{\ell}}{2 \ell+1}+4 \pi \sum_{m>0}\left|a_{\ell m}\right|^{2}\left\{2 \frac{\lambda_{\ell}}{2 \ell+1}-m\right\} \\
& =4 \pi \sum_{m=-\ell}^{\ell}\left|a_{\ell m}\right|^{2}\left\{\frac{\lambda_{\ell}}{2 \ell+1}-\frac{m}{2}\right\},
\end{aligned}
$$

and

$$
I_{22}(\ell)=\frac{4 \pi}{2} \sum_{m=-\ell}^{\ell}\left|a_{\ell m}\right|^{2} m
$$

(b) For the cross-product integral, we have that

$$
I_{0,22}(\ell)=-4 \pi a_{\ell 0}^{2} \frac{\ell}{2 \ell+1}+4 \pi \sum_{m>0}\left|a_{\ell m}\right|^{2}\left(\frac{1}{2 \ell+1}-m\right) .
$$

(c) Finally, for the remaining terms

$$
\begin{aligned}
I_{12,12}(\ell) & =4 \pi \sum_{m>0}\left|a_{\ell m}\right|^{2} m\left\{\frac{\lambda-1-m^{2}}{2}\right\} \\
& =4 \pi \sum_{m=-\ell}^{\ell}\left|a_{\ell m}\right|^{2} m\left\{\frac{\lambda-1-m^{2}}{4}\right\},
\end{aligned}
$$

and

$$
I_{22,22}(\ell)=4 \pi \frac{a_{\ell 0}^{2}}{2}\left(\ell^{2}-\frac{\ell}{2 \ell+1}\right)+\frac{4 \pi}{2} \sum_{m>0}\left|a_{\ell m}\right|^{2}\left\{-\frac{4 \lambda}{2 \ell+1}+m+\lambda m+m^{3}\right\} .
$$


PROOF. We introduce here the standard basis for spherical harmonics (see, e.g., [17], Section 13.2), which is given by

$$
Y_{\ell m}(\theta, \varphi)= \begin{cases}e^{i m \varphi} \sqrt{\frac{2 \ell+1}{4 \pi} \frac{(\ell-m) !}{(\ell+m) !}} P_{\ell}^{m}(\cos \theta), & m \geq 0, \\ (-1)^{m} e^{i m \varphi} \sqrt{\frac{2 \ell+1}{4 \pi} \frac{(\ell+m) !}{(\ell-m) !}} P_{\ell}^{-m}(\cos \theta), & m<0,\end{cases}
$$

where we introduced also the associated Legendre functions, which are defined by

$$
P_{\ell}^{m}(x)= \begin{cases}(-1)^{m}\left(1-x^{2}\right)^{m / 2} \frac{d^{m}}{d x^{m}} P_{\ell}(x), & m \geq 0, \\ (-1)^{m} \frac{(\ell+m) !}{(\ell-m) !} P_{\ell}^{-m}(x), & m<0 .\end{cases}
$$

Let us recall also the trivial orthogonality relationships

$$
\int_{0}^{2 \pi} e^{i m \varphi} e^{i n \varphi} d \varphi= \begin{cases}2 \pi, & n=-m \\ 0, & n \neq-m\end{cases}
$$

which yield

$$
\begin{aligned}
& \frac{1}{2 \ell+1} \int_{0}^{2 \pi} Y_{\ell m}(\theta, \varphi) Y_{\ell n}(\theta, \varphi) d \varphi \\
& \quad= \begin{cases}\frac{1}{2} \frac{(\ell-m) !}{(\ell+m) !}(-1)^{m}\left\{P_{\ell}^{m}(\cos \theta)\right\}^{2}, & n=-m \\
0, & n \neq-m\end{cases}
\end{aligned}
$$

Our next tool are the analytic expression for derivatives of spherical harmonics, which we recall to be given by

$e_{1}^{x} Y_{\ell m}(x)=\frac{\partial}{\partial \theta} Y_{\ell m}(\theta, \varphi), \quad e_{2}^{x} Y_{\ell m}(x)=\frac{1}{\sin \theta} \frac{\partial}{\partial \varphi} Y_{\ell m}(\theta, \varphi)=\frac{i m}{\sin \theta} Y_{\ell m}(\theta, \varphi)$,

and moreover,

$$
\begin{aligned}
e_{1}^{x} e_{2}^{x} Y_{\ell m}(x) & =\frac{1}{\sin \theta} \frac{\partial}{\partial \varphi} \frac{\partial}{\partial \theta} Y_{\ell m}(\theta, \varphi)-\frac{\cos \theta}{\sin ^{2} \theta} \frac{\partial}{\partial \varphi} Y_{\ell m}(\theta, \varphi) \\
& =\frac{i m}{\sin \theta} \frac{\partial}{\partial \theta} Y_{\ell m}(\theta, \varphi)-i m \frac{\cos \theta}{\sin ^{2} \theta} Y_{\ell m}(\theta, \varphi) \\
e_{2}^{x} e_{2}^{x} Y_{\ell m}(x) & =\frac{1}{\sin ^{2} \theta} \frac{\partial^{2}}{\partial \varphi^{2}} Y_{\ell m}(\theta, \varphi)+\frac{\cos \theta}{\sin \theta} \frac{\partial}{\partial \theta} Y_{\ell m}(\theta, \varphi) \\
& =-\frac{m^{2}}{\sin ^{2} \theta} Y_{\ell m}(\theta, \varphi)+\frac{\cos \theta}{\sin \theta} \frac{\partial}{\partial \theta} Y_{\ell m}(\theta, \varphi)
\end{aligned}
$$


Finally, we recall that the spherical harmonic coefficients satisfy the identities $(-1)^{m} a_{\ell,-m}=\bar{a}_{\ell m}$ ([17], formula (6.6)). The first part of (a) is then a trivial consequence of the Parseval's identity, or the orthonormality of spherical harmonics:

$$
I_{00}(\ell)=\int_{\mathbb{S}^{2}} f_{\ell}^{2}(x) d x=\frac{4 \pi}{2 \ell+1} \sum_{m=-\ell}^{\ell}\left|a_{\ell m}\right|^{2} .
$$

For the other two integrals in (a), the first step is to rewrite them as functions of derivatives of associated Legendre functions, as follows:

$$
\begin{aligned}
I_{11}(\ell)= & \int_{\mathbb{S}^{2}}\left\{e_{1}^{x} f_{\ell}(x)\right\}^{2} d x \\
= & 4 \pi \frac{a_{\ell 0}^{2}}{2} \int_{0}^{\pi}\left\{\frac{d}{d \theta} P_{\ell}^{0}(\cos \theta)\right\}^{2} \sin \theta d \theta \\
& +4 \pi \sum_{m>0}\left|a_{\ell m}\right|^{2} \frac{(\ell-m) !}{(\ell+m) !} \int_{0}^{\pi}\left\{\frac{d}{d \theta} P_{\ell}^{m}(\cos \theta)\right\}^{2} \sin \theta d \theta, \\
I_{22}(\ell)= & \int_{\mathbb{S}^{2}}\left\{e_{2}^{x} f_{\ell}(x)\right\}^{2} d x \\
= & 4 \pi \sum_{m>0}\left|a_{\ell m}\right|^{2} m^{2} \frac{(\ell-m) !}{(\ell+m) !} \int_{0}^{\pi} \frac{1}{\sin ^{2} \theta}\left\{P_{\ell}^{m}(\cos \theta)\right\}^{2} \sin \theta d \theta .
\end{aligned}
$$

The same approach is needed to rewrite the integral in (b):

$$
\begin{aligned}
I_{0,22}(\ell)= & \int_{\mathbb{S}^{2}} f_{\ell}(x) e_{2}^{x} e_{2}^{x} f_{\ell}(x) d x \\
= & -4 \pi \sum_{m>0}\left|a_{\ell m}\right|^{2} \frac{(\ell-m) !}{(\ell+m) !} m^{2} \int_{0}^{\pi} \frac{1}{\sin ^{2} \theta}\left\{P_{\ell}^{m}(\cos \theta)\right\}^{2} \sin \theta d \theta \\
& +4 \pi \frac{a_{\ell 0}^{2}}{2} \int_{0}^{\pi} \frac{\cos \theta}{\sin \theta} P_{\ell}^{0}(\cos \theta) \frac{d}{d \theta} P_{\ell}^{0}(\cos \theta) \sin \theta d \theta \\
& +4 \pi \sum_{m>0}\left|a_{\ell m}\right|^{2} \frac{(\ell-m) !}{(\ell+m) !} \int_{0}^{\pi} \frac{\cos \theta}{\sin \theta} P_{\ell}^{m}(\cos \theta) \frac{d}{d \theta} P_{\ell}^{m}(\cos \theta) \sin \theta d \theta,
\end{aligned}
$$

and similarly for (c):

$$
\begin{aligned}
I_{12,12}(\ell)= & \int_{\mathbb{S}^{2}}\left\{e_{1}^{x} e_{2}^{x} f_{\ell}(x)\right\}^{2} d x \\
= & 4 \pi \sum_{m>0}\left|a_{\ell m}\right|^{2} \frac{(\ell-m) !}{(\ell+m) !} m^{2}\left\{\int_{0}^{\pi} \frac{1}{\sin ^{2} \theta}\left\{\frac{d}{d \theta} P_{\ell}^{m}(\cos \theta)\right\}^{2} \sin \theta d \theta\right. \\
& \left.+\int_{0}^{\pi} \frac{\cos ^{2} \theta}{\sin ^{4} \theta}\left\{P_{\ell}^{m}(\cos \theta)\right\}^{2} \sin \theta d \theta\right\}
\end{aligned}
$$




$$
\begin{aligned}
& -2 \cdot 4 \pi \sum_{m>0}\left|a_{\ell m}\right|^{2} \frac{(\ell-m) !}{(\ell+m) !} m^{2} \\
& \times \int_{0}^{\pi} \frac{\cos \theta}{\sin ^{3} \theta}\left\{\frac{d}{d \theta} P_{\ell}^{m}(\cos \theta)\right\} P_{\ell}^{m}(\cos \theta) \sin \theta d \theta, \\
I_{22,22}(\ell)= & \int_{\mathbb{S}^{2}}\left\{e_{2}^{x} e_{2}^{x} f_{\ell}(x)\right\}^{2} d x \\
= & 4 \pi \sum_{m>0}\left|a_{\ell m}\right|^{2} \frac{(\ell-m) !}{(\ell+m) !} m^{4} \int_{0}^{\pi} \frac{1}{\sin ^{4} \theta}\left\{P_{\ell}^{m}(\cos \theta)\right\}^{2} \sin \theta d \theta \\
& +4 \pi \frac{a_{\ell 0}^{2}}{2} \int_{0}^{\pi} \frac{\cos ^{2} \theta}{\sin ^{2} \theta}\left\{\frac{d}{d \theta} P_{\ell}^{0}(\cos \theta)\right\}^{2} \sin \theta d \theta \\
& -2 \cdot 4 \pi \sum_{m>0}\left|a_{\ell m}\right|^{2} \frac{(\ell-m) !}{(\ell+m) !} m^{2} \\
& \times \int_{0}^{\pi} \frac{\cos \theta}{\sin ^{3} \theta} P_{\ell}^{m}(\cos \theta)\left\{\frac{d}{d \theta} P_{\ell}^{m}(\cos \theta)\right\} \sin \theta d \theta \\
& +4 \pi \sum_{m>0}\left|a_{\ell m}\right|^{2} \frac{(\ell-m) !}{(\ell+m) !} \int_{0}^{\pi} \frac{\cos { }^{2} \theta}{\sin ^{2} \theta}\left\{\frac{d}{d \theta} P_{\ell}^{m}(\cos \theta)\right\}^{2} \sin \theta d \theta .
\end{aligned}
$$

It is now convenient to introduce the following, more compact notation for integrals of associated Legendre functions and their derivatives; more precisely, we shall write

$$
\begin{aligned}
& J_{1}(\ell, m):=\frac{(\ell-m) !}{(\ell+m) !} \int_{0}^{\pi}\left\{\frac{d}{d \theta} P_{\ell}^{m}(\cos \theta)\right\}^{2} \sin \theta d \theta, \\
& J_{2}(\ell, m):=\frac{(\ell-m) !}{(\ell+m) !} \int_{0}^{\pi} \frac{1}{\sin ^{2} \theta}\left\{P_{\ell}^{m}(\cos \theta)\right\}^{2} \sin \theta d \theta, \\
& J_{3}(\ell, m):=\frac{(\ell-m) !}{(\ell+m) !} \int_{0}^{\pi} \frac{\cos \theta}{\sin \theta} P_{\ell}^{m}(\cos \theta) \frac{d}{d \theta} P_{\ell}^{m}(\cos \theta) \sin \theta d \theta, \\
& J_{4}(\ell, m):=\frac{(\ell-m) !}{(\ell+m) !} \int_{0}^{\pi} \frac{1}{\sin ^{2} \theta}\left\{\frac{d}{d \theta} P_{\ell}^{m}(\cos \theta)\right\}^{2} \sin \theta d \theta, \\
& J_{5}(\ell, m):=\frac{(\ell-m) !}{(\ell+m) !} \int_{0}^{\pi} \frac{\cos \theta}{\sin ^{3} \theta}\left\{\frac{d}{d \theta} P_{\ell}^{m}(\cos \theta)\right\} P_{\ell}^{m}(\cos \theta) \sin \theta d \theta, \\
& J_{6}(\ell, m):=\frac{(\ell-m) !}{(\ell+m) !} \int_{0}^{\pi} \frac{\cos ^{2} \theta}{\sin ^{4} \theta}\left\{P_{\ell}^{m}(\cos \theta)\right\}^{2} \sin \theta d \theta, \\
& J_{7}(\ell, m):=\frac{(\ell-m) !}{(\ell+m) !} \int_{0}^{\pi} \frac{1}{\sin ^{4} \theta}\left\{P_{\ell}^{m}(\cos \theta)\right\}^{2} \sin \theta d \theta,
\end{aligned}
$$


and

$$
J_{8}(\ell, m)=\frac{(\ell-m) !}{(\ell+m) !} \int_{0}^{\pi} \frac{\cos ^{2} \theta}{\sin ^{2} \theta}\left\{\frac{d}{d \theta} P_{\ell}^{m}(\cos \theta\}^{2} \sin \theta d \theta .\right.
$$

It is then readily verified that

$$
\begin{aligned}
I_{11}(\ell) & =4 \pi \frac{a_{\ell 0}^{2}}{2} J_{1}(\ell, 0)+4 \pi \sum_{m>0}\left|a_{\ell m}\right|^{2} J_{1}(\ell, m), \\
I_{22}(\ell) & =4 \pi \sum_{m>0}\left|a_{\ell m}\right|^{2} m^{2} J_{2}(\ell, m) ; \\
I_{0,22}(\ell) & =4 \pi \frac{a_{\ell 0}^{2}}{2} J_{3}(\ell, 0)+4 \pi \sum_{m>0}\left|a_{\ell m}\right|^{2}\left\{J_{3}(\ell, m)-m^{2} J_{2}(\ell, m)\right\}, \\
I_{12,12}(\ell) & =4 \pi \sum_{m>0}\left|a_{\ell m}\right|^{2} m^{2}\left\{J_{4}(\ell, m)-2 J_{5}(\ell, m)+J_{6}(\ell, m)\right\},
\end{aligned}
$$

and

$$
\begin{aligned}
I_{22,22}(\ell)= & 4 \pi \frac{a_{\ell 0}^{2}}{2} J_{8}(\ell, 0) \\
& +4 \pi \sum_{m>0}\left|a_{\ell m}\right|^{2}\left\{m^{4} J_{7}(\ell, m)-2 m^{2} J_{5}(\ell, m)+J_{8}(\ell, m)\right\} .
\end{aligned}
$$

The proof can then be completed by an explicit computation for the integrals $J_{a}(\ell, m), a=1, \ldots, 7$, which is given in Lemma 13 .

LEMMA 13. The following explicit evaluations hold for all $m=-\ell, \ldots, \ell$ :

$$
J_{1}(\ell, m)=2 \frac{\ell(\ell+1)}{2 \ell+1}-m
$$

for $m \neq 0$ we have

$$
J_{2}(\ell, m)=\frac{1}{m}, \quad J_{3}(\ell, m)=\frac{1}{2 \ell+1},
$$

and, for $m \neq 0, \pm 1$, we also have

$$
\begin{aligned}
& J_{4}(\ell, m)=\frac{m}{2} \frac{\ell^{2}+\ell+1-m^{2}}{m^{2}-1}, \\
& J_{8}(\ell, m)=\frac{1}{2}\left\{m+\frac{\ell(\ell+1)\left(4+m+2 \ell m-4 m^{2}\right)}{(2 \ell+1)\left(m^{2}-1\right)}\right\}, \\
& J_{5}(\ell, m)=\frac{\ell(\ell+1)}{2 m\left(m^{2}-1\right)}, \quad J_{6}(\ell, m)=\frac{\ell^{2}+\ell+1-m^{2}}{2 m\left(m^{2}-1\right)}, \\
& J_{7}(\ell, m)=\frac{\ell^{2}+\ell-1+m^{2}}{2 m\left(m^{2}-1\right)} .
\end{aligned}
$$


In particular, we note that, for all $m \neq 0$, the following identities hold:

$$
\begin{gathered}
J_{3}(\ell, m)-m^{2} J_{2}(\ell, m)=\frac{1}{2 \ell+1}-m, \\
J_{4}(\ell, m)-2 J_{5}(\ell, m)+J_{6}(\ell, m)=\frac{\ell(\ell+1)-m^{2}-1}{2 m}, \\
m^{4} J_{7}(\ell, m)-2 m^{2} J_{5}(\ell, m)+J_{8}(\ell, m) \\
=\frac{1}{2}\left\{-4 \frac{\ell(\ell+1)}{2 \ell+1}+m+\ell(\ell+1) m+m^{3}\right\},
\end{gathered}
$$

and that, for $m=0$, we have

$$
J_{1}(\ell, 0)=2 \frac{\ell(\ell+1)}{2 \ell+1}, \quad J_{3}(\ell, 0)=-\frac{2 \ell}{2 \ell+1}, \quad J_{8}(\ell, 0)=\ell^{2}-\frac{\ell}{2 \ell+1} .
$$

PROOF. The proofs are all easy consequences of some simple change of variables formulae and the analytic results on integrals of associated Legendre functions which we collected in Section 5.3. More precisely, exploiting Lemma 14, one obtains

$$
J_{2}(\ell, m)=\frac{(\ell-m) !}{(\ell+m) !} \int_{-1}^{1} \frac{1}{1-z^{2}}\left\{P_{\ell}^{m}(z)\right\}^{2} d z=\frac{1}{m},
$$

in view of (28) and (29), moreover, by applying (29), we have

$$
J_{6}(\ell, m)=\frac{(\ell-m) !}{(\ell+m) !} \int_{-1}^{1} \frac{z^{2}}{\left(1-z^{2}\right)^{2}}\left\{P_{\ell}^{m}(z)\right\}^{2} d z=\frac{\ell^{2}+\ell+1-m^{2}}{2 m\left(m^{2}-1\right)},
$$

and from (28)

$$
J_{7}(\ell, m)=\frac{(\ell-m) !}{(\ell+m) !} \int_{-1}^{1} \frac{1}{\left(1-z^{2}\right)^{2}}\left\{P_{\ell}^{m}(z)\right\}^{2} d z=\frac{\ell^{2}+\ell-1+m^{2}}{2 m\left(m^{2}-1\right)} .
$$

Similarly, from (27) we have

$$
J_{3}(\ell, m)=-\frac{(\ell-m) !}{(\ell+m) !} \int_{-1}^{1} z P_{\ell}^{m}(z) \frac{d}{d z} P_{\ell}^{m}(z) d z=\frac{1}{2 \ell+1},
$$

and, in view of Lemma 15 ,

$$
J_{5}(\ell, m)=-\frac{(\ell-m) !}{(\ell+m) !} \int_{-1}^{1} \frac{z}{1-z^{2}} P_{\ell}^{m}(z)\left\{\frac{d}{d z} P_{\ell}^{m}(z)\right\} d z=\frac{\ell(\ell+1)}{2 m\left(m^{2}-1\right)} .
$$

Finally, using Lemma 16, from (31) we have

$$
J_{4}(\ell, m)=\frac{(\ell-m) !}{(\ell+m) !} \int_{-1}^{1}\left\{\frac{d}{d z} P_{\ell}^{m}(z)\right\}^{2} d z=\frac{m}{2} \frac{\ell^{2}+\ell+1-m^{2}}{m^{2}-1} ;
$$


from (32) we have

$$
J_{1}(\ell, m)=\frac{(\ell-m) !}{(\ell+m) !} \int_{-1}^{1}\left(1-z^{2}\right)\left\{\frac{d}{d z} P_{\ell}^{m}(z)\right\}^{2} d z=2 \frac{\ell(\ell+1)}{2 \ell+1}-m,
$$

and, in view of (33),

$$
\begin{aligned}
J_{8}(\ell, m) & =\frac{(\ell-m) !}{(\ell+m) !} \int_{-1}^{1} z^{2}\left\{\frac{d}{d z} P_{\ell}^{m}(z)\right\}^{2} d z \\
& =\frac{1}{2}\left\{m+\frac{\ell(\ell+1)\left(4+m+2 \ell m-4 m^{2}\right)}{(2 \ell+1)\left(m^{2}-1\right)}\right\} .
\end{aligned}
$$

5.3. Some integrals of associated Legendre functions. In this final subsection, we need to report some explicit computations on integrals involving cross products of associated Legendre functions and their derivatives. For some of these results, we managed to find references, others may be known already but we failed to locate any suitable reference and, therefore, we report their proofs entirely; we believe they may have some independent interest for related works on the geometry of random spherical harmonics. In particular, the following two results are given in [28], equation (25) and equation (37), respectively,

$$
\begin{aligned}
\int_{-1}^{1} \frac{1}{1-z^{2}}\left\{P_{\ell}^{m}(z)\right\}^{2} d z & =\frac{(\ell+m) !}{m(\ell-m) !} \\
\int_{-1}^{1} z P_{\ell}^{m}(z)\left\{\frac{d}{d z} P_{\ell}^{m}(z)\right\} d z & =\delta_{0, m}-\frac{(\ell+m) !}{(2 \ell+1)(\ell-m) !} .
\end{aligned}
$$

The other integrals we shall need are given in the following three lemmas; the first deals with squares of associated Legendre functions, the second with cross-product of Legendre functions and their derivatives, the third with squared derivatives.

LEMMA 14. The following analytic expressions hold for all values of $\ell=$ $1,2,3, \ldots$ :

$$
\begin{aligned}
& \int_{-1}^{1} \frac{1}{\left(1-z^{2}\right)^{2}}\left\{P_{\ell}^{m}(z)\right\}^{2} d z \\
&= \frac{1}{4 m^{2}}\left\{\frac{(\ell+m)(\ell+m-1)(\ell+m) !}{(m-1)(\ell-m) !}+\frac{(\ell+m) !}{(m+1)(\ell-m-2) !}\right\} \\
& \int_{-1}^{1} \frac{z^{2}}{\left(1-z^{2}\right)^{2}}\left\{P_{\ell}^{m}(z)\right\}^{2} d z \\
&=\frac{1}{4 m^{2}}\left\{\frac{(\ell+m)(\ell+m-1)(\ell+m) !}{(m-1)(\ell-m) !}+\frac{(\ell+m) !}{(m+1)(\ell-m-2) !}\right\} \\
& \quad-\frac{(\ell+m) !}{m(\ell-m) !}
\end{aligned}
$$


PROOF. Formula (29) follows from (28) and (26):

$$
\begin{aligned}
\int_{-1}^{1} & \frac{z^{2}}{\left(1-z^{2}\right)^{2}}\left(P_{\ell}^{m}(z)\right)^{2} d z \\
& =\int_{-1}^{1} \frac{1}{\left(1-z^{2}\right)^{2}}\left(P_{\ell}^{m}(z)\right)^{2} d z-\int_{-1}^{1} \frac{1}{1-z^{2}}\left(P_{\ell}^{m}(z)\right)^{2} d z .
\end{aligned}
$$

To prove (28), we exploit the following identity (see, e.g., [16], Section 7.12):

$$
\frac{1}{\sqrt{1-z^{2}}} P_{\ell}^{m}(z)=-\frac{1}{2 m}\left[(\ell+m-1)(\ell+m) P_{\ell-1}^{m-1}(z)+P_{\ell-1}^{m+1}(z)\right]
$$

whence

$$
\begin{aligned}
& \int_{-1}^{1} \frac{1}{\left(1-z^{2}\right)^{2}}\left\{P_{\ell}^{m}(z)\right\}^{2} d z \\
&=\int_{-1}^{1} \frac{1}{1-z^{2}}\left\{\frac{P_{\ell}^{m}(z)}{\sqrt{1-z^{2}}}\right\}^{2} d z \\
&=\frac{1}{4 m^{2}} \int_{-1}^{1} \frac{1}{1-z^{2}}\left\{(\ell+m-1)(\ell+m) P_{\ell-1}^{m-1}(z)+P_{\ell-1}^{m+1}(z)\right\}^{2} d z \\
&=\frac{(\ell+m-1)^{2}(\ell+m)^{2}}{4 m^{2}} \int_{-1}^{1} \frac{1}{1-z^{2}}\left\{P_{\ell-1}^{m-1}(z)\right\}^{2} d z \\
&+\frac{(\ell+m-1)(\ell+m)}{2 m^{2}} \int_{-1}^{1} \frac{1}{1-z^{2}} P_{\ell-1}^{m-1}(z) P_{\ell-1}^{m+1}(z) d z \\
&+\frac{1}{4 m^{2}} \int_{-1}^{1} \frac{1}{1-z^{2}}\left\{P_{\ell-1}^{m+1}(z)\right\}^{2} d z ;
\end{aligned}
$$

the statement immediately follows by applying twice equation (26):

$$
\begin{aligned}
& \int_{-1}^{1} \frac{1}{1-z^{2}}\left\{P_{\ell-1}^{m-1}(z)\right\}^{2} d z=\frac{(\ell+m-2) !}{(m-1)(\ell-m) !} \\
& \int_{-1}^{1} \frac{1}{1-z^{2}}\left\{P_{\ell-1}^{m+1}(z)\right\}^{2} d z=\frac{(\ell+m) !}{(m+1)(\ell-m-2) !},
\end{aligned}
$$

and by observing that

$$
\int_{-1}^{1} \frac{1}{1-z^{2}} P_{\ell-1}^{m-1}(z) P_{\ell-1}^{m+1}(z) d z=0 .
$$

LEMMA 15. The following analytic expressions hold for all values of $\ell=$ $1,2,3, \ldots$ :

$$
\begin{aligned}
\int_{-1}^{1} & \frac{z}{1-z^{2}} P_{\ell}^{m}(z)\left\{\frac{d}{d z} P_{\ell}^{m}(z)\right\} d z \\
& =\frac{1}{4 m}\left\{\frac{(\ell+m+1) !}{(m+1)(\ell-m-1) !}-\frac{(\ell+m)(\ell-m+1)(\ell+m) !}{(m-1)(\ell-m) !}\right\} .
\end{aligned}
$$


PROOF. We first note that

$$
\begin{aligned}
z P_{\ell}^{m}(z) & =-\frac{\sqrt{1-z^{2}}}{2 m}\left[(\ell+m)(\ell-m+1) P_{\ell}^{m-1}(z)+P_{\ell}^{m+1}(z)\right], \\
\sqrt{1-z^{2}} \frac{d}{d z} P_{\ell}^{m}(z) & =\frac{1}{2}\left[(\ell+m)(\ell-m+1) P_{\ell}^{m-1}(z)-P_{\ell}^{m+1}(z)\right],
\end{aligned}
$$

so that

$$
\begin{aligned}
\int_{-1}^{1} \frac{z}{1-z^{2}} P_{\ell}^{m}(z)\left\{\frac{d}{d z} P_{\ell}^{m}(z)\right\} d z \\
=-\frac{1}{4 m} \int_{-1}^{1} \frac{1}{1-z^{2}}\left[(\ell+m)^{2}(\ell-m+1)^{2}\left\{P_{\ell}^{m-1}(z)\right\}^{2}-\left\{P_{\ell}^{m+1}(z)\right\}^{2}\right] d z \\
=-\frac{(\ell+m)^{2}(\ell-m+1)^{2}}{4 m} \int_{-1}^{1} \frac{1}{1-z^{2}}\left\{P_{\ell}^{m-1}(z)\right\}^{2} d z \\
\quad+\frac{1}{4 m} \int_{-1}^{1} \frac{1}{1-z^{2}}\left\{P_{\ell}^{m+1}(z)\right\}^{2} d z
\end{aligned}
$$

and, by applying (26), we immediately have the statement.

LEMMA 16. The following analytic expressions hold for all values of $\ell=$ $1,2,3, \ldots$ :

$$
\begin{aligned}
& \int_{-1}^{1}\left\{\frac{d}{d z} P_{\ell}^{m}(z)\right\}^{2} d z \\
& =\frac{1}{4}\left\{\frac{(\ell+m)(\ell-m+1)(\ell+m) !}{(m-1)(\ell-m) !}+\frac{(\ell+m+1) !}{(m+1)(\ell-m-1) !}\right\} \\
& \int_{-1}^{1}\left(1-z^{2}\right)\left\{\frac{d}{d z} P_{\ell}^{m}(z)\right\}^{2} d z
\end{aligned}
$$

$$
\begin{aligned}
& =\frac{1}{(2 \ell+1)^{2}}\left\{\frac{(\ell+1)^{2}(\ell+m)(\ell+m) !}{m(\ell-m-1) !}\right. \\
& \left.-2 \frac{\ell(\ell+1)(\ell-m+1)(\ell+m) !}{m(\ell-m-1) !}+\frac{\ell^{2}(\ell-m+1)^{2}(\ell+m+1) !}{m(\ell-m+1) !}\right\},
\end{aligned}
$$

$$
\int_{-1}^{1} z^{2}\left\{\frac{d}{d z} P_{\ell}^{m}(z)\right\}^{2} d z
$$

$$
=-\frac{1}{(2 \ell+1)^{2}}\left\{\frac{(\ell+1)^{2}(\ell+m)(\ell+m) !}{m(\ell-m-1) !}\right.
$$




$$
\begin{aligned}
& \left.-2 \frac{\ell(\ell+1)(\ell-m+1)(\ell+m) !}{m(\ell-m-1) !}+\frac{\ell^{2}(\ell-m+1)^{2}(\ell+m+1) !}{m(\ell-m+1) !}\right\} \\
& +\frac{1}{4}\left\{\frac{(\ell+m)(\ell-m+1)(\ell+m) !}{(m-1)(\ell-m) !}+\frac{(\ell+m+1) !}{(m+1)(\ell-m-1) !}\right\} .
\end{aligned}
$$

PROOF. To prove (31), we use

$$
\frac{d}{d z} P_{\ell}^{m}(z)=\frac{1}{2 \sqrt{1-z^{2}}}\left\{(\ell+m)(\ell-m+1) P_{\ell}^{m-1}(z)-P_{\ell}^{m+1}(z)\right\}
$$

so that we may write

$$
\begin{aligned}
\int_{-1}^{1}\left\{\frac{d}{d z} P_{\ell}^{m}(z)\right\}^{2} d z \\
=\frac{1}{4} \int_{-1}^{1} \frac{1}{1-z^{2}}\left\{(\ell+m)(\ell-m+1) P_{\ell}^{m-1}(z)-P_{\ell}^{m+1}(z)\right\}^{2} d z \\
=\frac{(\ell+m)^{2}(\ell-m+1)^{2}}{4} \int_{-1}^{1} \frac{1}{1-z^{2}}\left\{P_{\ell}^{m-1}(z)\right\}^{2} d z \\
\quad+\frac{1}{4} \int_{-1}^{1} \frac{1}{1-z^{2}}\left\{P_{\ell}^{m+1}(z)\right\}^{2} d z \\
\quad-\frac{(\ell+m)(\ell-m+1)}{2} \int_{-1}^{1} \frac{1}{1-z^{2}} P_{\ell}^{m-1}(z) P_{\ell}^{m+1}(z) d z
\end{aligned}
$$

Formula (31) then follows by observing that, from (26), we have

$$
\begin{gathered}
\frac{(\ell+m)^{2}(\ell-m+1)^{2}}{4} \int_{-1}^{1} \frac{1}{1-z^{2}}\left\{P_{\ell}^{m-1}(z)\right\}^{2} d z \\
=\frac{(\ell+m)^{2}(\ell-m+1)^{2}}{4} \frac{(\ell+m-1) !}{(m-1)(\ell-m+1) !},
\end{gathered}
$$

and

$$
\frac{1}{4} \int_{-1}^{1} \frac{1}{1-z^{2}}\left\{P_{\ell}^{m+1}(z)\right\}^{2} d z=\frac{1}{4} \frac{(\ell+m+1) !}{(m+1)(\ell-m-1) !}
$$

and moreover,

$$
\int_{-1}^{1} \frac{1}{1-z^{2}} P_{\ell}^{m-1}(z) P_{\ell}^{m+1}(z) d z=0 .
$$

To prove (32), we apply the following identity (see [16], Section 7.12):

$$
\left(1-z^{2}\right) \frac{d}{d z} P_{\ell}^{m}(z)=\frac{1}{2 \ell+1}\left\{(\ell+1)(\ell+m) P_{\ell-1}^{m}(z)-\ell(\ell-m+1) P_{\ell+1}^{m}(z)\right\}
$$


from which we obtain

$$
\begin{aligned}
\int_{-1}^{1}(1 & \left.-z^{2}\right)\left\{\frac{d}{d z} P_{\ell}^{m}(z)\right\}^{2} d z \\
= & \frac{1}{(2 \ell+1)^{2}} \int_{-1}^{1} \frac{1}{1-z^{2}} \\
& \times\left\{(\ell+1)(\ell+m) P_{\ell-1}^{m}(z)-\ell(\ell-m+1) P_{\ell+1}^{m}(z)\right\}^{2} d z \\
= & \frac{(\ell+1)^{2}(\ell+m)^{2}}{(2 \ell+1)^{2}} \int_{-1}^{1} \frac{1}{1-z^{2}}\left\{P_{\ell-1}^{m}(z)\right\}^{2} d z \\
& +\frac{\ell^{2}(\ell-m+1)^{2}}{(2 \ell+1)^{2}} \int_{-1}^{1} \frac{1}{1-z^{2}}\left\{P_{\ell+1}^{m}(z)\right\}^{2} d z \\
& -2 \frac{(\ell+1)(\ell+m) \ell(\ell-m+1)}{(2 \ell+1)^{2}} \int_{-1}^{1} \frac{1}{1-z^{2}} P_{\ell-1}^{m}(z) P_{\ell+1}^{m}(z) d z .
\end{aligned}
$$

Formula (32) follows by applying again (26), which gives

$$
\begin{aligned}
\int_{-1}^{1} \frac{1}{1-z^{2}}\left\{P_{\ell-1}^{m}(z)\right\}^{2} d z & =\frac{(\ell+m-1) !}{m(\ell-m-1) !}, \int_{-1}^{1} \frac{1}{1-z^{2}}\left\{P_{\ell+1}^{m}(z)\right\}^{2} \\
& =\frac{(\ell+m+1) !}{m(\ell-m+1) !},
\end{aligned}
$$

and [28], formula (24i), which gives

$$
\int_{-1}^{1} \frac{1}{1-z^{2}} P_{\ell-1}^{m}(z) P_{\ell+1}^{m}(z) d z=\frac{(\ell+m-1) !}{m(\ell-m-1) !} .
$$

Finally, to prove (33) it is sufficient to note that

$$
\begin{aligned}
\int_{-1}^{1} z^{2} & \left\{\frac{d}{d z} P_{\ell}^{m}(z)\right\}^{2} d z \\
= & -\int_{-1}^{1}\left(1-z^{2}\right)\left\{\frac{d}{d z} P_{\ell}^{m}(z)\right\}^{2} d z+\int_{-1}^{1}\left\{\frac{d}{d z} P_{\ell}^{m}(z)\right\}^{2} d z \\
= & -\frac{1}{(2 \ell+1)^{2}}\left\{\frac{(\ell+1)^{2}(\ell+m)(\ell+m) !}{m(\ell-m-1) !}-2 \frac{\ell(\ell+1)(\ell-m+1)(\ell+m) !}{m(\ell-m-1) !}\right. \\
& \left.+\frac{\ell^{2}(\ell-m+1)^{2}(\ell+m+1) !}{m(\ell-m+1) !}\right\} \\
& +\frac{1}{4}\left\{\frac{(\ell+m)(\ell-m+1)(\ell+m) !}{(m-1)(\ell-m) !}+\frac{(\ell+m+1) !}{(m+1)(\ell-m-1) !}\right\} .
\end{aligned}
$$

Acknowledgments. We are grateful to Giovanni Peccati, Maurizia Rossi and Igor Wigman for a number of insightful discussions; we are also grateful to an 
Associate Editor and three anonymous referees for comments and suggestions on an earlier version.

\section{REFERENCES}

[1] AdLER, R. J. and TAYLOR, J. E. (2007). Random Fields and Geometry. Springer, New York. MR2319516

[2] Adler, R. J. and TAYlor, J. E. (2011). Topological Complexity of Smooth Random Functions. Lecture Notes in Math. 2019. Springer, Heidelberg. Lectures from the 39th Probability Summer School held in Saint-Flour, 2009, École d'Été de Probabilités de SaintFlour. [Saint-Flour Probability Summer School]. MR2768175

[3] AzAïs, J.-M. and Wschebor, M. (2009). Level Sets and Extrema of Random Processes and Fields. Wiley, New York.

[4] Berry, M. V. (1977). Regular and irregular semiclassical wavefunctions. J. Phys. A $102083-$ 2091.

[5] Cammarota, V., Marinucci, D. and Wigman, I. (2016). On the distribution of the critical values of random spherical harmonics. J. Geom. Anal. 4 3252-3324.

[6] Cammarota, V., Marinucci, D. and Wigman, I. (2016). Fluctuations of the EulerPoincaré characteristic for random spherical harmonics. Proc. Amer. Math. Soc. 114759 4775.

[7] Cammarota, V. and Wigman, I. (2017). Fluctuations of the total number of critical points of random spherical harmonics. Stochastic Process. Appl. 127 3825-3869.

[8] CanZani, Y. and Hanin, B. (2015). Fixed frequency eigenfunction immersions and supremum norms of random waves.. Electron. Res. Announc. Math. Sci. 22 76-86.

[9] Chavel, I. (2006). Riemannian Geometry: A Modern Introduction, 2nd ed. Cambridge Studies in Advanced Mathematics 98. Cambridge Univ. Press, Cambridge. MR2229062

[10] Cheng, D. and XiaO, Y. (2016). Excursion probability of Gaussian random fields on sphere. Bernoulli 22 1113-1130. MR3449810

[11] Cheng, D. and Xiao, Y. (2016). The mean Euler characteristic and excursion probability of Gaussian random fields. Ann. Appl. Probab. 26 722-759. MR3476623

[12] Dalmao, F., Nourdin, I., Peccati, G. and Rossi, M. (2016). Phase singularities in complex arithmetic random waves. Preprint. Available at arXiv:1608.05631.

[13] Dehling, H. and TAQQu, M. (1989). The empirical process of some long-range dependent sequences with an application to U-statistics. Ann. Statist. 17 1767-1783. MR1026312

[14] Estrade, A. and León, J. R. (2016). A central limit theorem for the Euler characteristic of a Gaussian excursion set. Ann. Probab. 44 3849-3878. MR3572325

[15] Krishnapur, M., Kurlberg, P. and Wigman, I. (2013). Nodal length fluctuations for arithmetic random waves. Ann. of Math. 177 699-737.

[16] Lebedev, N. N. (1965). Special Functions and Their Applications. Prentice-Hall, Inc., Englewood Cliffs, N.J.

[17] MarinucCi, D. and PeCCATI, G. (2011). Random Fields on the Sphere: Representation, Limit Theorems and Cosmological Applications. London Mathematical Society Lecture Note Series 389. Cambridge Univ. Press, Cambridge. MR2840154

[18] Marinucci, D., Peccati, G., Rossi, M. and Wigman, I. (2016). Non-universality of nodal length distribution for arithmetic random waves. Geom. Funct. Anal. 26 926-960. MR3540457

[19] MarinuCCI, D. and Rossi, M. (2015). Stein-Malliavin approximations for nonlinear functionals of random eigenfunctions on $S^{d}$. J. Funct. Anal. 268 2379-2420.

[20] MarinucCi, D., Rossi, M. and Wigman, I. (2017). The asymptotic equivalence of the sample trispectrum and the nodal length for random spherical harmonics. Preprint. Available at arXiv:1705.05747. 
[21] Marinucci, D. and Vadlamani, S. (2016). High-frequency asymptotics for LipschitzKilling curvatures of excursion sets on the sphere. Ann. Appl. Probab. 26 462-506. MR3449324

[22] MarinuCCI, D. and Wigman, I. (2011). On the area of excursion sets of spherical Gaussian eigenfunctions. J. Math. Phys. 52093301.

[23] MARINUCCI, D. and Wigman, I. (2014). On nonlinear functionals of random spherical eigenfunctions. Comm. Math. Phys. 327 849-872. MR3192051

[24] NAZARov, F. and Sodin, M. (2009). On the number of nodal domains of random spherical harmonics. Amer. J. Math. 131 1337-1357.

[25] Nourdin, I. and PeCCATI, G. (2012). Normal Approximations with Malliavin Calculus: From Stein's Method to Universality. Cambridge Tracts in Mathematics 192. Cambridge Univ. Press, Cambridge. MR2962301

[26] Peccati, G. and Rossi, M. (2016). Quantitative limit theorems for local functionals of arithmetic random waves. Preprint. Available at arXiv:1702.03765.

[27] Rossi, M. (2015). The geometry of spherical random fields. Ph.D. thesis, Univ. Rome Tor Vergata. Available at arXiv:1603.07575.

[28] Samaddar, S. N. (1974). Some integrals involving associated Legendre functions. Math. Comp. 28 257-263.

[29] Sarnak, P. and Wigman, I. (2015). Topologies of nodal sets of random band limited functions. Preprint. Available at arXiv:1510.08500.

[30] TAYlor, J. E. (2006). A Gaussian kinematic formula. Ann. Probab. 34 122-158. MR2206344

[31] TAYLOR, J. E. and VAdLAMANI, S. (2013). Random fields and the geometry of Wiener space. Ann. Probab. 41 2724-2754.

[32] Wigman, I. (2010). Fluctuations of the nodal length of random spherical harmonics. Comm. Math. Phys. 298 787-831.

[33] Wigman, I. (2012). On the nodal lines of random and deterministic Laplace eigenfunctions. In Spectral Geometry. Proc. Sympos. Pure Math. 84 285-297. AMS, Providence. Available at arXiv:1103.0150.

DiPARTIMENTO Di SCIENZE STATISTICHE

UNIVERSITÀ DEGLI STUDI DI ROMA

'LA SAPIENZA'

PiazZAle Aldo Moro, 5

ROMA, 00185

ITALY

E-MAIL: valentina.cammarota@uniroma1.it
DiPARTIMENTO Di MATEMATICA

UNIVERSITÀ DEGLI STUDI DI ROMA

'TOR VERGATA'

VIA DELla RICERCA SCIENTIFICA 1

ROMA, 00133

ITALY

E-MAIL: marinucc@mat.uniroma2.it 